\title{
White matter microstructural and morphometric alterations in autism: Implications for intellectual and verbal capabilities
}

\author{
Chun-Hung Yeh ${ }^{1,2, *}$,a , Rung-Yu Tseng ${ }^{1,2, a}$, Hsing-Chang $\mathrm{Ni}^{2}$, Luca Cocchi ${ }^{3}$, Jung-Chi Chang ${ }^{4}$, Mei- \\ Yun $\mathrm{Hsu}^{5}$, En-Nien Tu${ }^{6,7}$, Yu-Yu Wu ${ }^{5}$, Tai-Li Chou ${ }^{8}$, Susan Shur-Fen Gau ${ }^{4}$, Hsiang-Yuan Lin ${ }^{4,9,10, *}$ \\ ${ }^{1}$ Institute for Radiological Research, Chang Gung University and Chang Gung Memorial Hospital, Taoyuan, \\ Taiwan \\ ${ }^{2}$ Department of Psychiatry, Chang Gung Memorial Hospital at Linkou, Taoyuan, Taiwan \\ ${ }^{3}$ Clinical Brain Networks Group, QIMR Berghofer Medical Research Institute, Brisbane, Queensland, Australia \\ ${ }^{4}$ Department of Psychiatry, National Taiwan University Hospital and College of Medicine, Taipei, Taiwan \\ ${ }^{5}$ YuNing Clinic, Taipei, Taiwan \\ ${ }^{6}$ Department of Psychiatry, University of Oxford, Oxford, United Kingdom \\ ${ }^{7}$ Department of Psychiatry, Keelung Chang Gung Memorial Hospital, Keelung, Taiwan \\ ${ }^{8}$ Department of Psychology, National Taiwan University, Taipei, Taiwan \\ ${ }^{9}$ Azrieli Adult Neurodevelopmental Centre, Campbell Family Mental Health Research Institute, and Adult \\ Neurodevelopmental and Geriatric Psychiatry Division, Centre for Addiction and Mental Health, Toronto, \\ Ontario, Canada \\ ${ }^{10}$ Department of Psychiatry and Institute of Medical Science, Temerty Faculty of Medicine, University of \\ Toronto, Toronto, Ontario, Canada \\ ${ }^{\mathrm{a}}$ These authors contributed equally to this work as co-first authors.
}

*Correspondence to:

Hsiang-Yuan Lin

Address:

Azrieli Adult Neurodevelopmental Centre, Centre for Addiction and Mental Health 1025 Queen St West - 3314, Toronto, ON, Canada M6J 1H4.

Email: $\quad$ Hsiang-Yuan.Lin@ camh.ca; hsiangyuan.lin@utoronto.ca

Chun-Hung Yeh

Address: Institute for Radiological Research, Chang Gung University.

No. 259, Wenhua 1st Road, Guishan District, 333 Taoyuan City, Taiwan

Email: $\quad$ jimmy.chyeh@gmail.com; chunhung.yeh@ mail.cgu.edu.tw

Contents: Abstract 250 words. Main text: 4479 words; 1 table; 4 figures; Supplement: Supplementary methods, supplementary results, 1 supplementary table; 6 supplementary figures. 


\section{ABSTRACT}

\section{Background}

Neuroimage literature of autism spectrum disorder (ASD) has a moderate-to-high risk of bias, partially because those combined with intellectual impairment (II) and/or minimally verbal (MV) status are generally ignored. We aimed to provide more comprehensive insights into white matter alterations of ASD, inclusive of individuals with II (ASD-II-Only) or MV expression (ASD-MV).

\section{Methods}

Sixty-five participants with ASD (ASD-Whole; 16.6 \pm 5.9 years; comprising 34 intellectually able youth, ASD-IA, and 31 intellectually impaired youth, ASD-II, including 24 ASD-II-Only plus 7 ASD-MV) and 38 demographic-matched typically developing controls (TDC; 17.3 \pm 5.6 years) were scanned in accelerated diffusion-weighted MRI. Fixel-based analysis was undertaken to investigate the categorical differences in fiber density (FD), fiber cross-section (FC), and a combined index (FDC), and brainsymptom/cognition associations.

\section{Results}

ASD-Whole had reduced FD in the anterior and posterior corpus callosum and left cerebellum Crus I, and smaller FDC in right cerebellum Crus II, compared to TDC. ASD-II, relative to TDC, showed almost identical alterations to those from ASD-Whole vs. TDC. ASD-II-Only had greater FD/FDC in the isthmus-splenium of callosum than ASD-MV. Autistic severity negatively correlated with FC in right Crus I. Non-verbal full-scale IQ positively correlated with FC/FDC in cerebellum VI. FD/FDC of the right dorsolateral prefrontal cortex showed a diagnosis-by-executive function interaction.

\section{Conclusions}

ASD-associated white matter alterations appear driven by individuals combined with II and/or MV.

Results suggest that changes in the corpus callosum and cerebellum are key for psychopathology and cognition associated with ASD. Our work highlights an essential to include understudied subpopulations on the spectrum in research.

Keywords: Autism spectrum disorder, fixel-based analysis, intellectual disabilities, minimally verbal status, cerebellum, diffusion MRI 


\section{INTRODUCTION}

Autism Spectrum Disorder (ASD) is a neurodevelopmental condition, characterized by struggling in socio-communication and the restricted/repetitive behaviors and interests (American Psychiatric Association, 2013). ASD is associated with categorically and dimensionally neurodevelopmental alterations in brain structures and function, contributing to suboptimal information processing underpinning social-communication, sensorimotor integration, and executive control processes (Anagnostou and Taylor, 2011, Rommelse et al., 2017).

Supporting this observation and extending this understanding to the level of brain connections, studies using diffusion magnetic resonance imaging (dMRI) showed that altered white matter (WM) microstructural properties associated with ASD exist in WM tracts interconnecting brain regions/systems involved in social processing and executive control (Ameis and Catani, 2015, Aoki et al., 2013, Di et al., 2018, Travers et al., 2012). For example, increased mean diffusivity (MD) or decreased fractional anisotropy (FA) in the corpus callosum (CC), uncinate fasciculus, superior longitudinal fasciculus, and frontal and temporal thalamic projections have been reported in people with ASD. However, high variability of findings is commonly noted across studies, partially because of limitations and factors that can affect dMRI results. Specifically, voxel-based analysis (VBA) and tractography of dMRI popularly involve the analysis based on the diffusion tensor model (Basser et al., 1994). But this model fails to resolve the multiple fiber orientations in regions containing crossing fibers, which exists in most WM voxels (Jeurissen et al., 2013). Thus, advanced dMRI models to address this methodological challenge are crucial to advance understanding of the WM pathology associated with ASD.

The state-of-the-art fixel-based analysis, FBA (Tournier et al., 2019), provides the fiber tractspecific analysis to estimate the quantitative metrics associated with a single fiber population within a voxel (called 'fixel'), as opposed to analyses of voxel-averaged metrics. FBA has been shown to be 
more sensitive and interpretable than voxel-wise methods (Mito et al., 2018, Raffelt et al., 2017b), in terms of better reflection of the local WM features and morphology, by providing quantitative information of each fiber bundle cross-section given multiple crossing fibers within a voxel. Recently, the FBA has been applied to dMRI studies on ASD (Dimond et al., 2019, Kirkovski et al., 2020), showing promising but mixed results. Specifically, Dimond et al. (2019) reported that able youth with ASD have lower microstructural density in the right uncinate and arcuate fasciculi alongside CCsplenium, and splenium fixel density (FD; defined in Methods) is associated with social impairment. Kirkovski et al. (2020) nevertheless only observed altered micro-/macro-structure in the posterior midbody of CC in able female adults with ASD. This inconsistency may, in part, reflect different sampling protocols of dMRI (e.g., number of gradient directions and b-values, resulting in variations of diffusion signals (Afzali et al., 2021) across studies, as well as heterogeneity among individuals with ASD. Moreover, both studies only focused on individuals with relatively intact language and cognitive functioning, limiting the representativeness of the autistic cohort.

In addition to the above methodological considerations, around 50\% of children with ASD have cognitive and intellectual impairments (i.e., IQ scores <85, representing intellectual disabilities (ID) plus borderline intelligence (Maenner et al., 2020)), and about 30\% of children with ASD remain minimally verbal (MV) by reaching their school age (Tager-Flusberg and Kasari, 2013). Unfortunately, these "low-functioning" individuals are understudied and generally excluded from neuroimaging research. Despite prominent heterogeneity in designs, samples and findings, results from the limited existing studies suggest that people with ASD and ID have alterations of gray matter (GM) and WM morphology in diffuse regions implicated in ASD pathology (Jack and Pelphrey, 2017). Specifically, children with MV ASD showed structural disruptions in language pathways (Jack and Pelphrey, 2017, Tager-Flusberg and Kasari, 2013). The paucity of studies including individuals with co-occurring 
developmental disabilities (Jack and Pelphrey, 2017) do, however, bias the understanding of brain bases of ASD (Happe and Frith, 2020).

Using the state-of-the-art dMRI acquisition (four b-values with 200 gradient directions), data processing framework (FBA), as well as the extensive psychopathological measures, the current study aimed to provide more comprehensive insights into the structural brain changes underpinning ASD. The current study included individuals with ID and MV, who are generally left out in the current lore. We hypothesized that the FBA could highlight potential differences in ASD that are driven by intellectual challenges. Based on previous work, we expected that intellectually able individuals with ASD would show alterations of WM fibers in the CC (Dimond et al., 2019, Kirkovski et al., 2020), uncinate and superior longitudinal fasciculi, and the thalamic radiation. Individuals with ASD plus cognitive impairment (including those with MV expression) would show alterations with broader spatial involvement. Autistic traits, cognitive impairment, and poor adaptive function were in fact expected to map onto fixel pathology in the tract interconnecting social and cognitive brain networks.

\section{METHODS}

\section{Procedures and Participants}

The study was approved by the Research Ethics Committee of National Taiwan University Hospital (\#201512238RINC). Participants with ASD (aged 8-30 years) were recruited from the outpatient psychiatric clinic at National Taiwan University Hospital, Chang Gung Memorial Hospital, Linkou, and Yuning Clinic. Age-matched TDC were recruited from neighborhoods with a similar social-economic environment to the ASD group. Before implementing the experiment, for those capable of giving consent themselves (i.e., showing capacity to understand the protocol), written 
informed consent was obtained from each participant and their parents. For those who were incapable of consenting, assent was sought and their substitute decision-maker signed the informed consent.

Eighty-six participants with a clinical diagnosis of ASD, made by child psychiatrists based on the DSM-5, and 39 TDC initially joined the study. The ASD diagnosis in all participants in the ASD group were further confirmed by the Autism Diagnostic Observation Schedule, ADOS (Chen et al., 2019, Lord et al., 2000) and Autism Diagnostic Interview-Revised, ADI-R (Gau et al., 2013, Rutter et al., 2003).

Parents of all participants received an interview by the senior author (H.-Y.L.) using the KiddieSchedule for Affective Disorders and Schizophrenia-Epidemiological Version (K-SADS-E) for DSM-5 (Chen et al., 2017), to evaluate co-occurring psychiatric disorders in patients and to ensure that TDC were free of any mental health issues. Exclusion criteria for all participants in the ASD group included: Any acute or unstable medical illness; history of psychosurgery or head trauma; any active grand mal seizures in the past one year; known genetic causes contributing to ASD or ID; a history of bipolar, psychotic or substance use disorders; current depressive symptoms or suicidal ideation; pregnancy.

In addition to clinical assessments, all participants received the intellectual assessments using the Wechsler Intelligence Scale-4 ${ }^{\text {th }}$ edition, either WAIS-IV (Wechsler, 2008) or WISC-IV (Wechsler and Kodama, 2003) with the age cutoff of 16 years, and Leiter International Performance Scale-Revised, Leiter-R (Roid and Miller, 2002). Their parents completed several scales to estimate participants' behaviors and function, including the Social Responsiveness Scales, SRS (Gau et al., 2013), for autistic traits, the Vineland Adaptive Behavior Scales, VABS (Lu, 1993, Sparrow et al., 1984), for adaptive function (adaptive behavior composite), and the Behavior Rating Inventory of Executive Function, BRIEF (Gioia et al., 2000), for daily-life executive function.

After the quality control steps detailed as follows, 65 participants with ASD (6 females, age: 16.6 \pm 5.9 years) and 38 TDC ( 8 females, age: $17.3 \pm 5.6$ years $)$ were included in the final dMRI analysis 
(Table 1). Among those excluded, 14 participants with ASD failed to complete the dMRI scan; 7 participants with ASD and 2 TDC exhibited excessive motion during the dMRI scan.

The whole ASD group (hereinafter ASD-Whole) was categorized into two subgroups: Intellectually Able (hereinafter ASD-IA; those with Wechsler's full-scale IQ, FSIQ, and adaptive behavior composite $>85, \mathrm{n}=34$ ) and Intellectual Impairment (hereinafter ASD-II; those with adaptive function and/or FSIQ <85, 1.5 standard deviations lower from the TDC norm; $\mathrm{n}=31$ ). The cutoff for "Intellectual Impairment" was defined because autistic children with borderline IQ (i.e., 70-84) have similar developmental trajectories to those combined with intellectual disabilities (Olsson et al., 2017). Adaptive function was included in the definition, because intelligence alone is imprecise to predict functional abilities of autistic individuals (Alvares et al., 2020). Moreover, we intentionally did not adopt the terms "high/low-functioning" herein to preclude prejudicial and inaccurate descriptions (Alvares et al., 2020, Den Houting, 2019).

In the ASD-II subgroup, there were 24 individuals with intellectual and cognitive impairment but fair verbal expression capacity (hereinafter ASD-II-Only); 15 out of these 24 ASD-II-Only had FSIQ<70. The other 7 participants in ASD-II had an MV status (hereinafter ASD-MV; defined by the effective use of $<30$ words in verbal expression as reported by their parents (Tager-Flusberg and Kasari, 2013)).

Seven participants with ASD received ADOS Module 1; four received Module 2; twenty-four received Module 3; and thirty received Module 4. We further transformed an ADOS-2 algorithm total raw score (Gotham et al., 2007) to a standardized calibrated severity score (CSS) (Gotham et al., 2009, Hus and Lord, 2014) to allow for the cross-module analysis.

\section{MRI Acquisition and Data Preprocessing}

MRI data were collected on a Siemens MAGNETOM Prisma 3-T MRI with a 64-channel phased-array head/neck coil. High-resolution MPRAGE T1-weighted structural brain images were 
acquired sagittally using the following parameters: repetition time $(\mathrm{TR})=2000 \mathrm{~ms}$, echo time $(\mathrm{TE})=2.43$ $\mathrm{ms}$, inversion time $=920 \mathrm{~ms}$, flip angle $=9^{\circ}$, acquisition matrix $=256 \times 256$, slice thickness $=0.9 \mathrm{~mm}$, inplane resolution=0.9 $\mathrm{mm}$ isotropic. Multi-shell diffusion-weighted imaging (DWI) data were acquired using the multi-band accelerated echo-planar imaging sequence developed at CMRR (Xu et al., 2013) with the following parameters: 2.2-mm isotropic voxel, TR/TE=2238/86 ms, multi-band acceleration factor=4, number of diffusion gradient directions $=19 / 30 / 90 / 60$ at $b=0 / 350 / 1000 / 3000 \mathrm{~s} / \mathrm{mm}^{2}$, respectively. Additional $b=0$ images with opposing phase encoding polarity were acquired for the correction of image distortion and motion (as described below).

DWI data pre-processing included denoising (Veraart et al., 2016), Gibbs ringing removal (Kellner et al., 2016), corrections for image distortions induced by eddy currents and susceptibility effects, inter-volume and slice-to-volume movements (Andersson et al., 2018, Andersson et al., 2017, Andersson and Sotiropoulos, 2016), bias field (Tustison et al., 2010). Quality assessments were performed to exclude the raw DWI data with excessive signal loss, artifacts, or in-scanner motion (based on the average root-mean-square displacements between DWI volumes, relative RMS, >1mm). The preprocessed DWI data of 38 TDC and 65 autistic participants were up-sampled and eventually analyzed (Table 1) using FBA described in the next section with recommended pipeline and parameters (Tournier et al., 2019). We also conducted complementary analysis, using the metrics FA and MD based on the diffusion tensor model, to investigate how specific the FBA results were (detailed in Supplement).

\section{Metrics and Statistics of FBA}

The multi-shell multi-tissue constrained spherical deconvolution was applied on each upsampled DWI to compute fiber orientation distributions (FODs) of WM and tissue compartments of GM and cerebrospinal fluid (Jeurissen et al., 2014), followed by intensity normalization to correct for compartmental inhomogeneities (Raffelt et al., 2017a). A study-specific FOD template was created 
from all TDC and ASD-Whole participants using the FOD-guided registration (Raffelt et al., 2011), followed by FOD segmentation (Smith et al., 2013) to generate the template fixels (FOD threshold=0.06). All participants' fixel-wise measures were computed and mapped onto the corresponding template fixels. The fixel-wise metrics include: FD, measuring microstructural fixelwise fiber density of specific WM fiber bundles within voxels; fiber cross-section (FC), measuring macrostructural change of a fiber bundle; a combined measure of FD and FC (FDC), quantifying the overall connectivity (micro-/macro-structure) per fiber bundle at the fixel level (detailed interpretations in (Raffelt et al., 2017b)).

We performed the statistical analysis of whole-brain fixel-wise metrics using the general linear model (GLM) incorporated with the connectivity-based fixel enhancement (CFE) approach (Raffelt et al., 2015). A whole-brain tractogram was generated using the template FODs, post-processed using spherical-deconvolution informed filtering of tractograms (SIFT) (Smith et al., 2013), and then used to compute fixel-to-fixel connectivity required for fixel data smoothing and enhanced statistics (Raffelt et al., 2015). Two types of analyses were conducted as follows:

- Categorical analysis - As described previously, our study cohort includes two main groups (TDC and ASD-Whole) and four ASD subgroups (ASD-IA, ASD-II, ASD-II-Only, and ASD-MV) subdivided from the ASD-Whole. Whole-brain fixel-wise differences were tested between pairs of these groups, including TDC versus each ASD (sub)group, and additionally, ASD-IA versus ASD-II and ASD-II-Only versus ASD-MV.

- Dimensional brain-behavior analysis - Mass-univariate GLMs for the whole sample (ASDWhole plus TDC) were separately constructed to investigate which fixel (dependent variable) in the brain could be predicted by each of the following independent variables, including non-verbal IQ (NVFIQ) using the Leiter-R, adaptive behavior composite, the SRS total raw score, and global executive composite (GEC) on the BRIEF. A behavior-by-diagnosis (TDC vs. ASD) interaction 
term was also included in these models. Notably, Wechsler's IQ was not used here because its assessment demands basic verbal proficiency, which results in the failure of individuals in the ASD-MV subgroup to complete the test. Another consideration was worth noting: Although the SRS manual provides T-scores for clinical screening use, the raw score is commonly used in research to estimate impairment in social functioning in people with ASD (Prigge et al., 2018). In addition, within the ASD-Whole group, we also undertook a mass-univariate GLM to investigate the brain-behavior relationship with an independent variable of ADOS-2 CSS.

For both analyses above, the nuisance variables included participant's sex, age, medication, ICV, and relative RMS. Considering the potential age effects on behavior (Taylor and Seltzer, 2010) and brain metrics (Bedford et al., 2020, Lin et al., 2015), we also additionally tested whether there were correlations between the age and aforementioned behavior/cognition variables, as well as whether there was an age diagnosis effect on FBA metrics. All these age-related tests yielded null results. The familywise error (FWE) corrected $P$-value (hereinafter $P$-FWE) was assigned to each fixel using nonparametric permutation testing over 5,000 permutations. The significant level was defined at $P$ $F W E<0.05$. To identify the associated WM anatomy with fixels, we used TractSeg to produce labeled WM fiber bundles (Wasserthal et al., 2018). When an identified fixel was located close to the GM or there were no major WM tracts nearby, we applied the cerebral AAL atlas (Tzourio-Mazoyer et al., 2002) and the cerebellar SUIT atlas (Diedrichsen, 2006) to obtain neuroanatomical labels. Yeo's 7network parcellation (Buckner et al., 2011, Yeo et al., 2011) was used to designate the functional organization to which these fibers belong. 


\section{RESULTS}

\section{Demographics}

As shown in Table 1, all three groups (TD, ASD-IA and ASD-II) had comparable distributions of sex, age, relative RMS, and intracranial volume. The ratio of medication uses and co-occurring psychiatric disorders were similar between ASD-IA and ASD-II (detailed in Table S1). The ASD-IA and TDC groups were matched for intellectual function measured by Wechsler's and Leiter's batteries. The ASD-IA group had intermediate levels of autistic symptoms, executive function, and overall adaptive function in-between the ASD-II and TDC groups.

Table 1. Demographic data and clinical features of participants

\begin{tabular}{|c|c|c|c|c|c|c|c|c|}
\hline & \multicolumn{2}{|c|}{$\begin{array}{c}\text { Typically Developing } \\
\text { Control (TDC) } \\
n=38\end{array}$} & \multicolumn{2}{|c|}{$\begin{array}{c}\text { Intellectually Able } \\
\text { ASD (ASD-IA) } \\
n=34 \\
\end{array}$} & \multicolumn{2}{|c|}{$\begin{array}{c}\text { Intellectually Impaired } \\
\text { ASD (ASD-II) } \\
n=31 \\
\end{array}$} & \multirow[t]{2}{*}{ P-value } & \multirow[t]{2}{*}{ Post-hoc test } \\
\hline & Mean & $\mathrm{SD}$ & Mean & $\mathrm{SD}$ & Mean & SD & & \\
\hline $\operatorname{Sex}(M: F)$ & \multicolumn{2}{|c|}{$30: 8$} & \multicolumn{2}{|c|}{$30: 4$} & \multicolumn{2}{|c|}{$29: 2$} & $0.20^{\mathrm{a}}$ & - \\
\hline Age (years) & 17.3 & 5.65 & 15.9 & 5.46 & 17.3 & 6.41 & $0.55^{\mathrm{b}}$ & - \\
\hline RMS (mm) & 0.399 & 0.197 & 0.407 & 0.184 & 0.463 & 0.216 & $0.40^{\mathrm{c}}$ & - \\
\hline Medication (Y:N) & \multicolumn{2}{|c|}{$0: 38$} & \multicolumn{2}{|c|}{$14: 20$} & \multicolumn{2}{|c|}{$9: 22$} & $0.31^{\mathrm{a}}$ & $-{ }^{\mathrm{d}}$ \\
\hline Comorbidity (Y:N) & \multicolumn{2}{|c|}{$0: 38$} & \multicolumn{2}{|c|}{$26: 8$} & \multicolumn{2}{|c|}{$24: 7$} & $0.93^{\mathrm{a}}$ & $-{ }^{d}$ \\
\hline $\mathrm{ICV}\left(\mathrm{cm}^{3}\right)$ & 1604.7 & 142.1 & 1588.8 & 114.5 & 1626.2 & 104.7 & $0.47^{\mathrm{b}}$ & - \\
\hline \multicolumn{2}{|c|}{$\begin{array}{l}\text { Cognition and Symptoms } \\
\text { FSIO }\end{array}$} & 11.7 & 104.5 & 14.2 & 65.7 & 13.0 & $<0.001^{\mathrm{c}}$ & ASD-II $<$ ASD-IA, TD \\
\hline NVFIQ (Leiter-R) & 121.6 & 10.2 & 115.0 & 18.3 & 77.6 & 26.3 & $<0.001^{\mathrm{c}}$ & ASD-II < ASD-IA, TD \\
\hline VABS-ABC & 113.5 & 15.3 & 89.2 & 15.1 & 66.7 & 9.6 & $<0.001^{\mathrm{c}}$ & ASD-II $<$ ASD-IA $<$ TD \\
\hline BRIEF-GEC & 92.3 & 19.1 & 155.3 & 28.5 & 152.5 & 25.5 & $<0.001^{\mathrm{c}}$ & ASD-II, ASD-IA > TD \\
\hline SRS-Total & 18.0 & 10.4 & 87.6 & 27.0 & 93.8 & 23.3 & $<0.001^{\mathrm{c}}$ & ASD-II $>$ ASD-IA $>$ TD \\
\hline ADOS-2 CSS & N/A & N/A & 5.0 & 2.1 & 6.8 & 2.1 & $0.002^{\mathrm{c}}$ & ASD-II $>$ ASD-IA ${ }^{d}$ \\
\hline
\end{tabular}

${ }^{\mathrm{a}}$ : Chi-square; ${ }^{\mathrm{b}}$ : ANOVA; ${ }^{\mathrm{c}}$ : Kruskal-Wallis; ${ }^{\mathrm{d}}$ : comparison between ASD-IA and ASD-II

Acronyms: RMS: relative Root-Mean-Square of framewise displacement during diffusion MRI; ICV: IntraCranial Volume; FSIQ: Full-Scale Intelligence Quotient; WISC-IV: Wechsler Intelligence Scale for Children4th edition; WAIS-IV: Wechsler Adult Intelligence Scale-4th edition; NVFIQ: Non-Verbal Intelligence Quotient; Leiter-R: Leiter International Performance Scale-Revised; VABS-ABC: Vineland Adaptive Behavior Scales 
Adaptive Behavior Composite; BRIEF-GEC: Behavior Rating Inventory of Executive Function Global Executive Composite; SRS-Total: Social Responsiveness Scales Total Raw Score; ADOS-2 CSS: Autism Diagnostic Observation Schedule 2 Calibrated Severity Score.

\section{Categorical Comparisons}

The ASD-Whole group, relative to the TDC, had smaller FD in the premotor segment and splenium of CC, alongside the left cerebellum Crus I (default-mode network), and smaller FDC in the right cerebellum Crus II (frontoparietal network; Figure 1, top row). Compared to the TDC group, ASD-II had smaller FD in the anterior (interconnecting bilateral prefrontal cortex) and posterior (part of the isthmus-splenium), and showed smaller FDC in the CC segment interconnecting bilateral premotor cortex, as well as in the right Crus II (frontoparietal network; Figure 1, middle row). Within the ASD-II subgroup, participants with ASD-II-Only, relative to ASD-MV, had greater FD in the middle segment and isthmus-splenium of CC. Similar to the FD result, the ASD-II-Only subgroup had greater FDC in the isthmus-splenium of CC than the ASD-MV subgroup (Figure 1, bottom row). There was no statistically significant difference in FBA metrics between the TDC and ASD-IA groups, or between the ASD-IA and ASD-II groups.

Given the spatial overlap, we extracted mean FD values from the common area of CC-splenium shared between ASD-Whole vs. TDC and ASD-II vs. TDC. Figure S1 illustrates distributions of this FD which showed that ASD-IA had a higher mean and smaller standard deviation of FD $(0.728 \pm 0.036)$ than ASD-Whole $(0.725 \pm 0.043)$ and ASD-II $(0.722 \pm 0.050)$, respectively. Together with its smaller sample size than that of ASD-Whole, this data feature result in null statistically significant differences between ASD-IA and TDC, alongside ASD-II, respectively (see detailed interpretations in Supplement). 


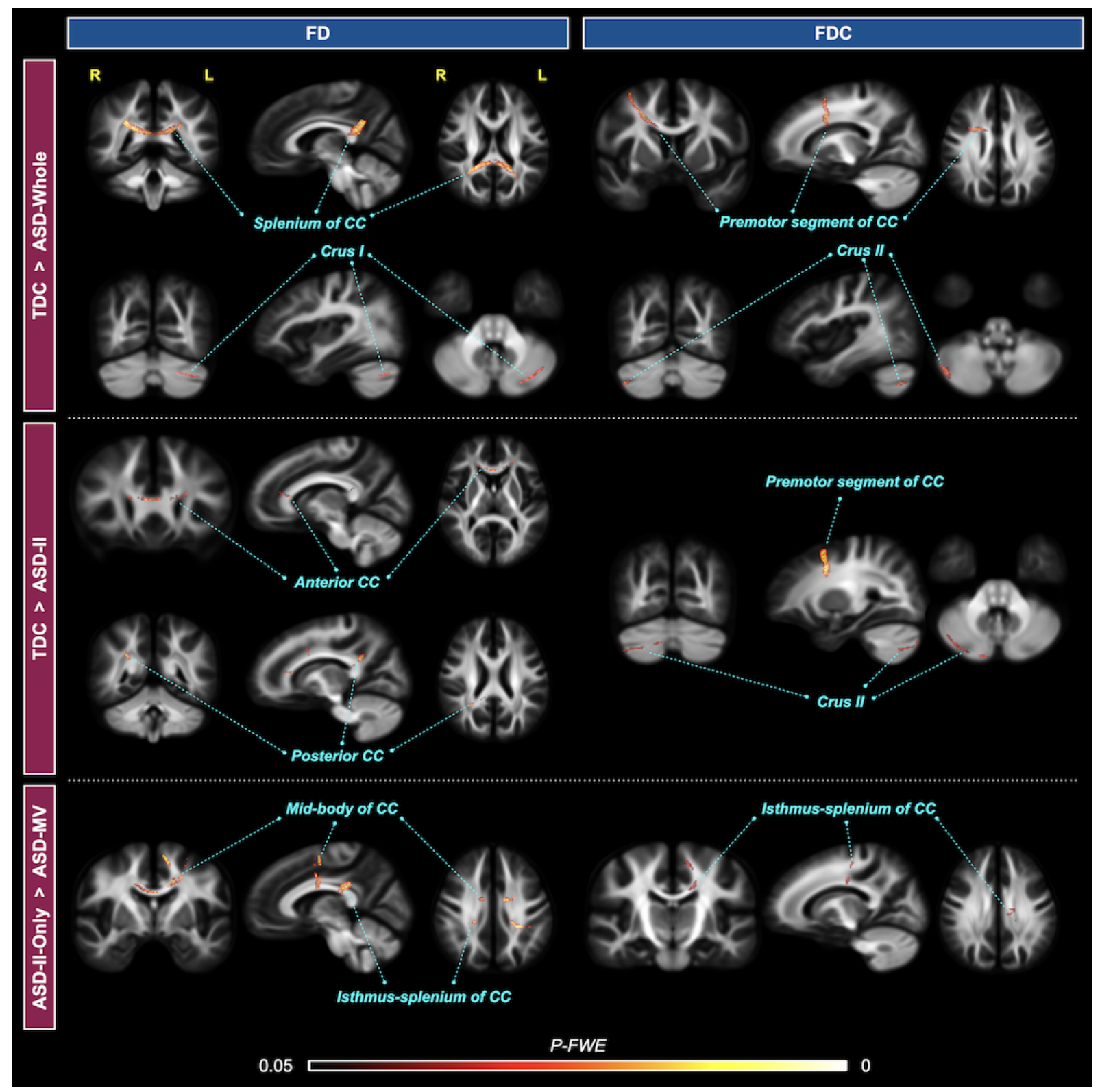

Figure 1: Results of categorical comparisons from the fixel-based analysis. White matter tract segments that have significant group differences in FD (left column) and FDC (right column) metrics are displayed and colored by the family-wise error corrected $P$-value $(P-F W E)$. Upper block - TDC > ASD; middle block - TDC > ASD-II; bottom block - ASD-II-Only > ASD-MV.

\section{Dimensional Brain-Behavior Relationship}

From the whole-sample GLMs, Figure 2 shows that NVFIQ significantly positively correlated with FC (Figure 2a-d) and FDC (Figure 2e-h) of fiber bundles in the cerebellum lobule VI (ventral 
attention/salience network. Significant GEC $\times$ diagnosis interactions were identified at the fibers at the GM-WM borders of the right dorsolateral prefrontal cortex (DLPFC, middle frontal gyrus subregion belonging to ventral attention/salience network; Figure 3a-b for FD, 3e-g for FDC). Specifically, GEC positively correlated with the DLPFC FD/FDC in TDC, while this brain-behavior association was negative in the ASD-Whole group (Figure 3d\&h). We did not find significant correlations or diagnosis $\times$ behavior interactions of the SRS and VABS with fixel metrics.

The within-ASD GLM yielded that autistic individuals' ADOS-2 CSS negatively correlated with WM FC in the right cerebellum Crus I (default-mode network; Figure 4a-d). Figure 4f shows cerebellar FA values are low ( 0.2), which cannot serve meaningful analysis. Figure 4e\&g illustrate that the cerebellar FBA FODs constituted a distinct structural organization that statistical analysis can be based upon. 


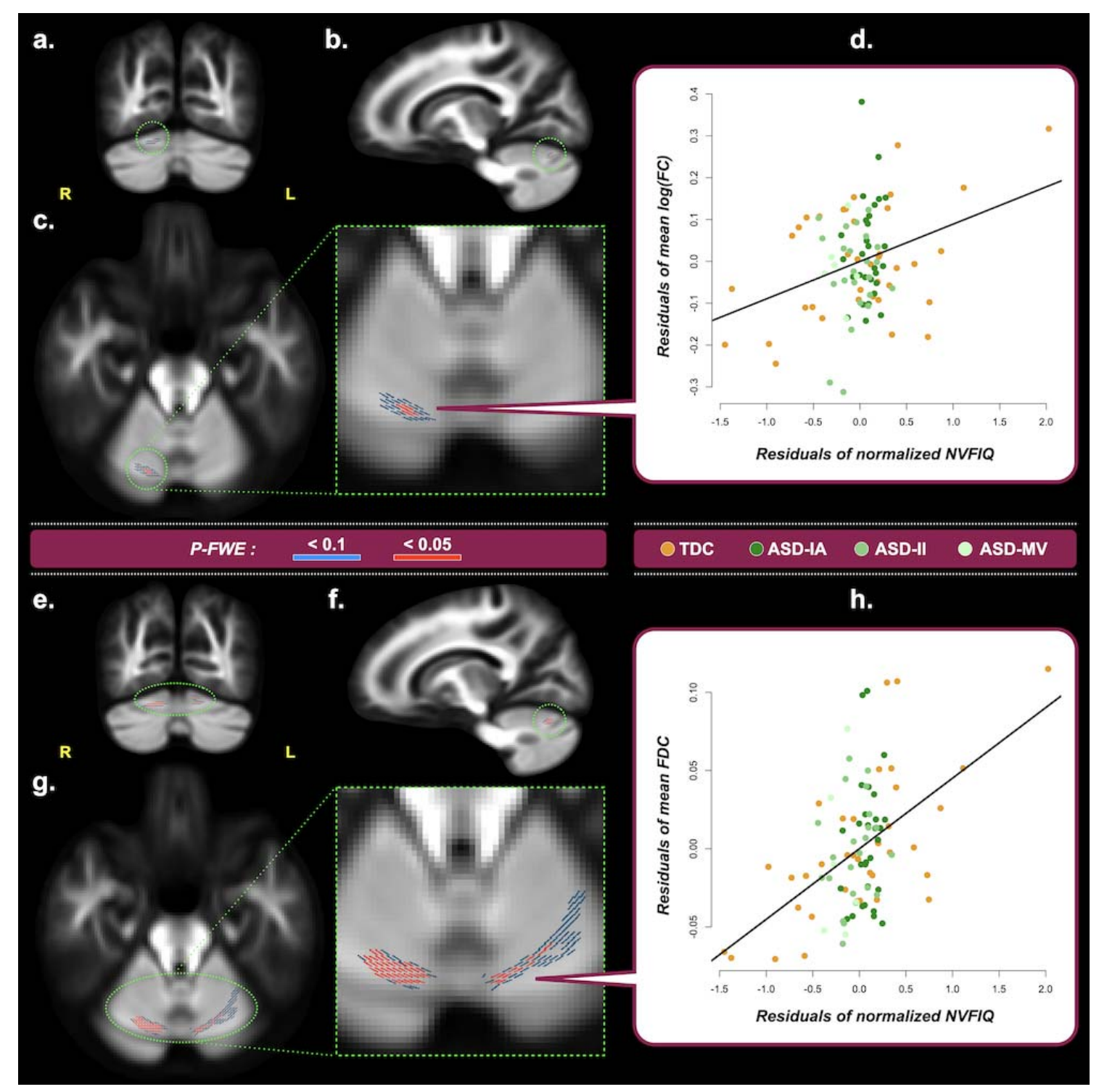

Figure 2: Correlations between fixel metrics and NVFIQ, derived from the dimensional analysis of the entire study cohort. Upper block - Panels a-c show the fixels where the correlation of $\log (\mathrm{FC})$ and Leiter's non-verbal full-scale IQ (NVFIQ) reach statistical significance from the coronal, sagittal, and transverse view, respectively. A zoomed image of (c) is displayed with the green dashed border. Fixels are colored in red for $P-F W E<0.05$; fixels colored in blue indicate $P-F W E<0.1$ and are used to assist identification of the associated brain structure. (d) The scatter plot shows the residuals of the mean $\log (\mathrm{FC})$ on the vertical axis and of the normalized NVFIQ on the horizontal axis. Only all fixels that reach $P-F W E<0.05$ are considered in the plot. Lower block - The format in Panels e-h is the same as the upper block, except that the results are obtained from the analysis of FDC and NVFIQ. Acronyms R: right; L: left; $P$-FWE: family-wise error corrected $P$-value. 

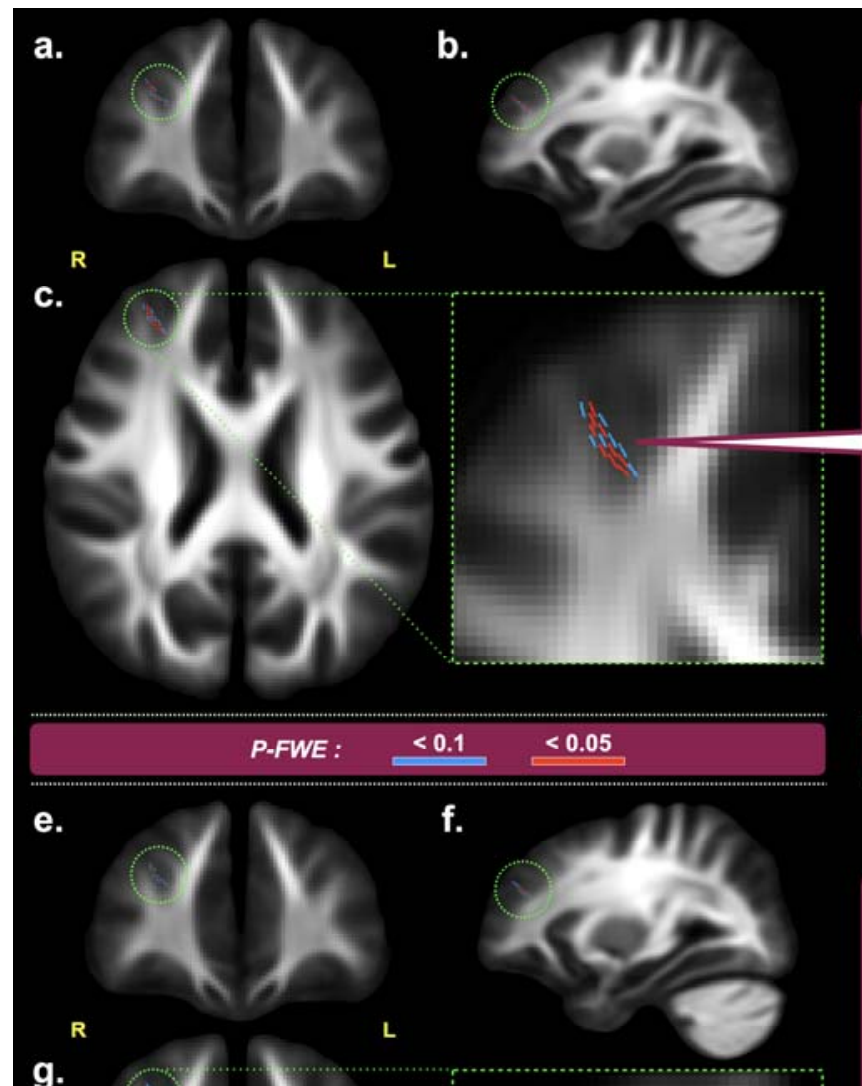

g.

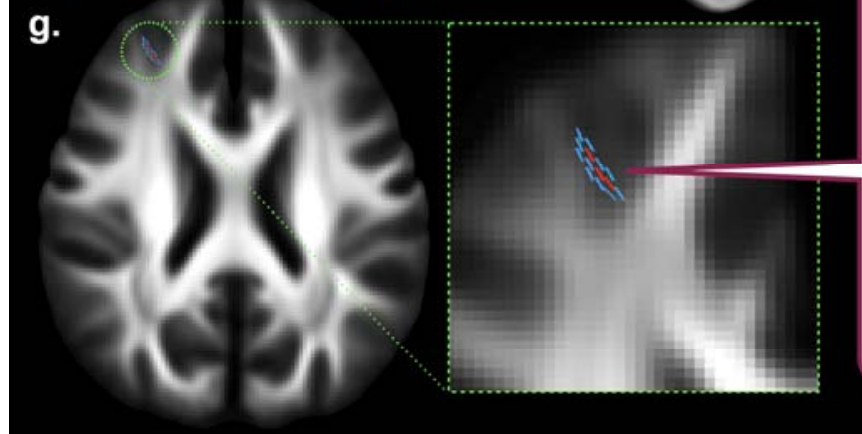

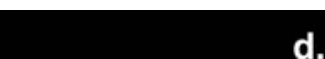

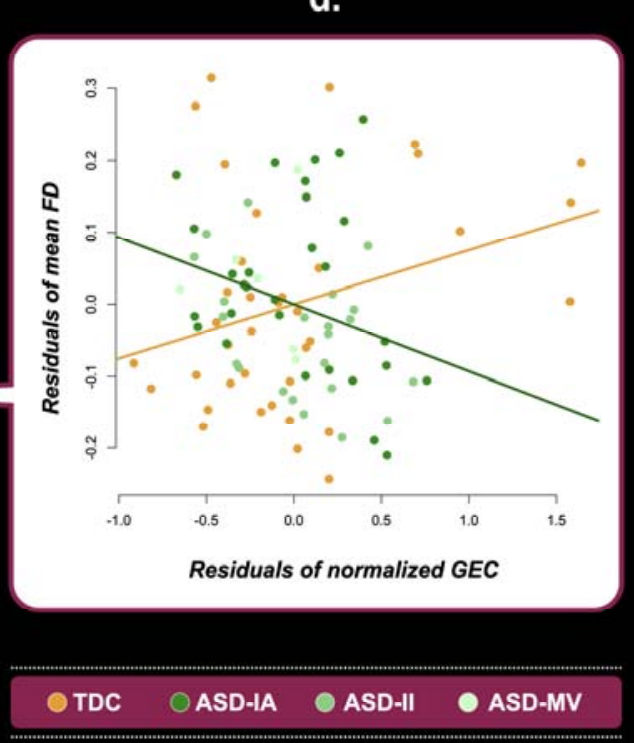

h.

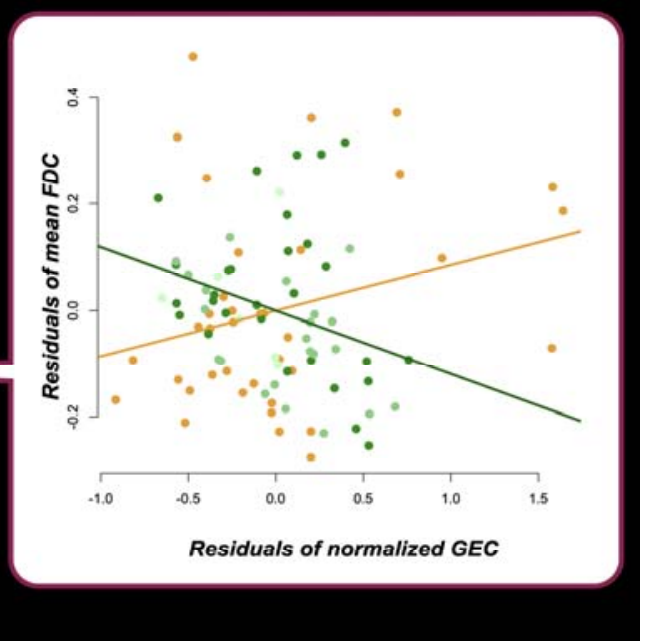

Figure 3: Correlations between fixel metrics and Global Executive Composite (GEC), derived from the dimensional analysis of the entire study cohort. Upper block - Panels a-c show the fixels where the correlation of FD and GECxdiagnosis interactions reach statistical significance from the coronal, sagittal, and transverse view, respectively. A zoomed image of (c) is displayed with the green dashed border. Fixels are colored in red for $P-F W E<0.05$; fixels colored in blue indicate $P-F W E<0.1$ and are used to assist identification of the associated brain structure. (d) The scatter plot shows the residuals of the mean FD on the vertical axis and of the normalized GEC on the horizontal axis. Only all fixels that reach $P-F W E<0.05$ are considered in the plot. Lower block - The format in Panels e-h is the same as the upper block, except that the results are obtained from the analysis of FDC and GEC. Acronyms - R: right; L: left; $P-F W E$ : family-wise error corrected $P$-value. 


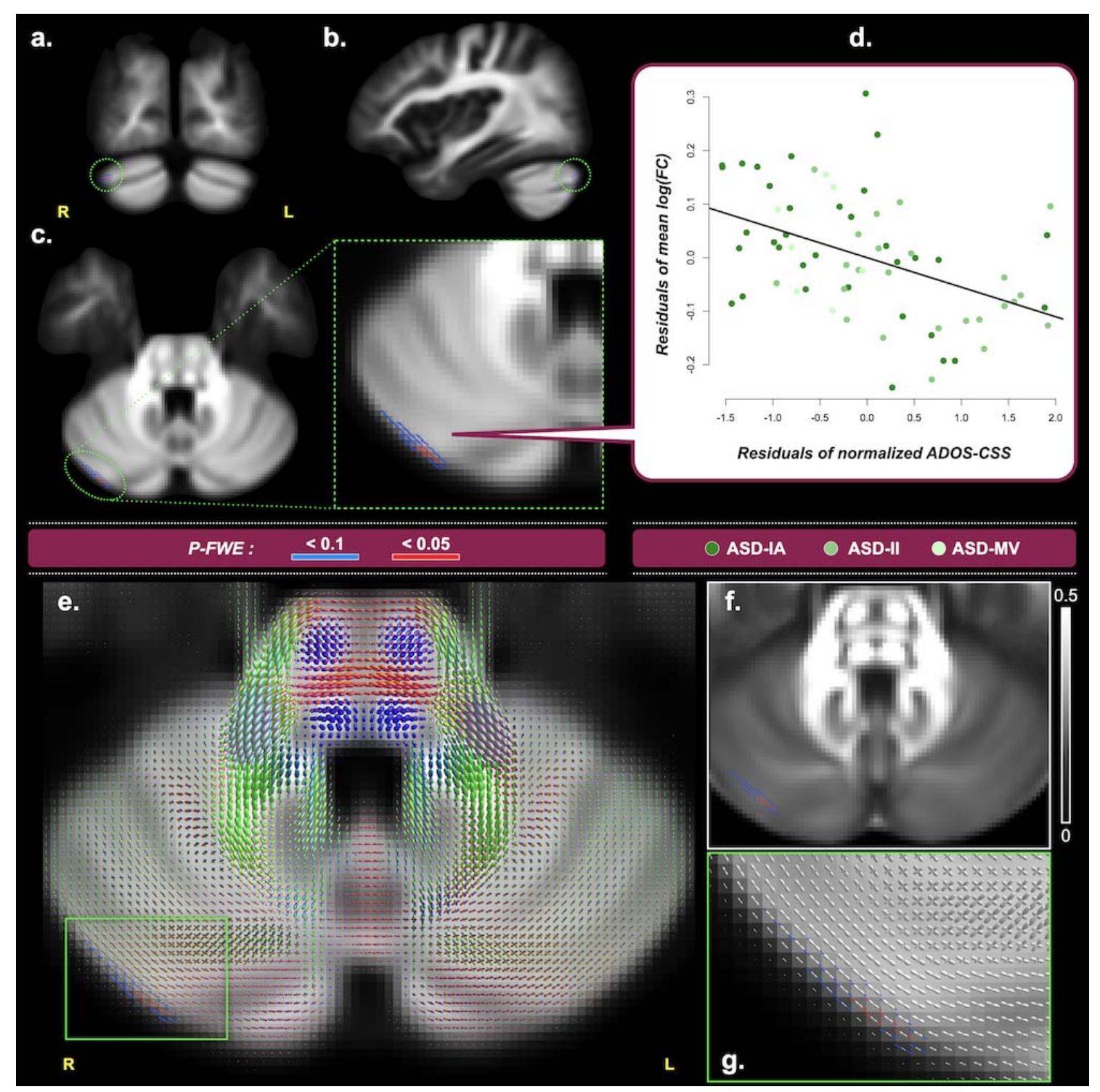

Figure 4: Correlations between fixel metrics and Autism Diagnostic Observation Schedule 2 Calibrated Severity Score (ADOS-CSS), derived from the dimensional analysis of the entire ASD cohort (i.e. ASD-Whole). Upper block - Panels a-c show the fixels where the correlation of $\log (\mathrm{FC})$ and ADOS-CSS reach statistical significance from the coronal, sagittal, and transverse view, respectively. A zoomed image of (c) is displayed with the green dashed border. Fixels are colored in red for $P-F W E<0.05$; fixels colored in blue indicate $P-F W E<0.1$ and are used to assist identification of the associated brain structure. (d) The scatter plot shows the mean $\log (\mathrm{FC})$ residuals on the vertical axis and the normalized ADOS-CSS residuals on the horizontal axis. Only all fixels that reach $P-F W E<0.05$ are considered in the plot. Lower block - (e) shows an axial slice of the group average FOD image. (f) shows the FA map of the same slice of (e). (g) is a zoomed region of (e), showing the microstructural 
organization of axonal fibers around the significant fixels within the cerebellum, which could not be reliably studied using tensor-based voxel-based analysis. Acronyms - R: right; L: left; $P$-FWE: familywise error corrected $P$-value.

\section{Robustness Testing}

This study adopted three strategies to test the robustness and specificity of current findings. First, during the generation of the template fixels, thresholding the amplitude of the FOD lobe is required to minimize the misalignment of fixels across subjects; such an effect is particularly pronounced at the GM-WM interface where registration could be imperfect (Raffelt et al., 2015). Hence, to ensure that the results were not driven by the choice of the FOD threshold, three additional FOD cutoff values of $0.04,0.08$, and 0.10 were used to construct the fixel template, and all the subsequent analyses including categorial comparisons and dimensional analysis were repeated. The main results in the categorical analysis above remained largely the same across different FOD thresholds (Figure S2). The repeated dimensional analysis also showed consistent findings in the correlated brain structures, despite some variations in fixel locations (Figure S3). Second, a more stringent significance level of $P-F W E<0.01$ was applied to test the current results. Few of the results met this strict criterion and they had reduced spatial extent of fixels (Figures S4-5). Lastly, VBA was performed with all the analyses repeated based on FA and MD. VBA neither replicated any of the results obtained from FBA nor provided additional findings (Figure S6).

\section{DISCUSSION}

Using the current state-of-the-art dMRI sequence and FBA in a cohort with ASD across the functional spectrum, we found that ASD-associated alterations in fixel-based metrics, as identified in ASD-Whole vs. TDC, were largely driven by those combined with intellectual impairment and/or 
minimally verbal status (Figure S1). In addition to the altered CC, consistent with earlier literature (Dimond et al., 2019, Travers et al., 2012), we provide initial evidence suggesting that cerebellar WM also categorically and dimensionally links with the clinical presentation of ASD. Our findings highlight an essential to include autistic participants with wide-range functional levels in research, to enable a greater a greater neurobiological understanding of the variety of functioning and cognitive profiles that manifest across the autism spectrum.

The present categorical comparison between ASD-Whole and TDC identified reduced FD in the splenium section of the $\mathrm{CC}$ and reduced FDC in the premotor segment of the $\mathrm{CC}$, consistent with an earlier FBA report on intellectually able individuals with ASD (Dimond et al., 2019). These alterations of the CC fibers are compatible with previous dMRI (Travers et al., 2012) and other modalities (Just et al., 2007, Paul et al., 2007) literature suggesting impaired interhemispheric connection might contribute to idiosyncratic socio-emotional behaviors and sensorimotor integration associated with ASD. In addition, we identified the ASD-Whole group showed altered geometries of fiber-bundle populations in the cerebellum Crus I/II. Moreover, autistic symptoms based on the ADOS correlated with the fiber bundle morphometry in the right Crus I. The left-side Crus I/II supports the socioemotional inferences and motion-related imitation (Arnold Anteraper et al., 2019, Jack and Pelphrey, 2015), whereas the right counterpart subserves the complex socio-emotional reasoning and sensorimotor integration (D'Mello and Stoodley, 2015, Siciliano and Clausi, 2020) in neurotypical people. The GM morphometry and function of Crus I/II are reported to be involved in impaired socioemotional (Siciliano and Clausi, 2020) and language processes, alongside repetitive behaviors (D'Mello and Stoodley, 2015) in people (van der Heijden et al., 2021) and mice models (Kelly et al., 2020, Stoodley et al., 2017) with ASD. Intellectually able youth with ASD also have specific developmental changes in GM morphometry at these loci (Lin et al., 2015, Siciliano and Clausi, 2020). Moreover, the identified Crus I and Crus II regions are separately functionally located in the default-mode and 
frontoparietal networks, of which altered cerebral activity and connectivity have been observed in ASD (Lin et al., 2019, Padmanabhan et al., 2017). Taken together, consistent with the functional MRI literature, we provide the first empirical evidence to imply that Crus I/II WM essentially links with autistic conditions. However, whether cerebellar lateralization plays a role in ASD-associated fiber pathology awaits further confirmation.

When comparing ASD-IA with TDC, with the similar sample size and demographic features to the participants of Dimond et al. (2019)'s work, we did not replicate the significant ASD-associated WM differences. Conversely, the ASD-II vs. TDC differences were similar to ASD-Whole vs. TDC differences in the CC-splenium (based on FD) and right Crus II (based on FDC). The difference between ASD-II-Only and ASD-MV, despite a small sample size, also was located at the posterior CC. As shown in Figure S1, our findings suggest that ASD-associated alterations in the geometry of fiberbundle populations, as identified by ASD-Whole vs. TDC, are mainly driven by the fiber pathology in those autistic people with lower intellectual/adaptive function. This uncovering might help reconcile parts of inconsistency in the previous FBA (Dimond et al., 2019, Kirkovski et al., 2020) and other MRI literature (Bernhardt et al., 2017). This is because these neuroimaging studies chiefly based on intellectually able individuals (Jack and Pelphrey, 2017) tend to show smaller effect sizes (Rodgaard et al., 2019) and be susceptible to sampling heterogeneity (Mottron and Bzdok, 2020). Our results also highlight the imperativeness of having research practices be inclusive of all autistic individuals on the spectrum, especially the understudied population with developmental disabilities, in order to address a gap of better characterizing these diverse autistic samples and development of more individualistic targeted plan accordingly (Happe and Frith, 2020, Jack and Pelphrey, 2017, Tager-Flusberg and Kasari, 2013).

The dimensional brain-behavior analysis showed that the positive correlation between NVFIQ and FC/FDC metrics in the cerebellum lobe VI existed cutting across ASD and TDC as a continuum. 
The higher the non-verbal intelligence is, the stronger the fiber-bundle populations are. Earlier functional MRI studies reported that the cerebellar VI is involved in motor processing, and nonmotor cognitive processing is implicated in language and working memory in ASD (Crucitti et al., 2020, D'Mello et al., 2015, Guell and Schmahmann, 2020, Guell et al., 2018). This posterolateral cerebellar area also supports the foundational function of hierarchical cognitive control such as mental arithmetic (King et al., 2019). This lobule also participates in the ventral attention/salience network (Habas et al., 2009), whose cerebral connectivity links with neurotypical individuals' fluid reasoning capacity (Yuan et al., 2012). Taken together, the current result suggests the essential role of the posterior cerebellum in contributing to human cognition, regardless of the neurodiverse status.

Moreover, we identified a diagnosis-specific relationship between the summarized measure of daily-life executive function and the FC/FDC in the GM-WM borders of the right DLPFC in the ventral attention/salience network. Individuals with ASD usually have a broad executive dysfunction that is relatively stable across development (Demetriou et al., 2018). The DLPFC is one of the primary areas that support executive function processing such as planning and working memory (Curtis and D'Esposito, 2003, Kobayashi, 2009). This prefrontal area plays an essential role in executive dysfunction associated with ASD across ages (Zhang et al., 2020). Moreover, ventral attention/salience network appears to link with ASD (Uddin, 2015) and self-control (Steimke et al., 2017) in different directions. ASD has poor delineation of the GM-WM boundary at the DLPFC (Andrews et al., 2017, Casanova, 2014). Collectively, while future studies are required to establish the relationship between the fiber-bundle populations and the differentiation of the GM-WM boundary, it is likely that altered neurodevelopmental processes involving WM fibers at the GM-WM boundary contribute to executive dysfunction commonly observed in ASD.

The capability of FBA to quantify fiber-specific measures of micro-/macro-structural alterations has been shown to be more beneficial for cerebral WM over VBA (Honnedevasthana Arun et al., 2021, 
Mito et al., 2018). Our study reveals that the cerebellum and the WM underneath as well as the GMWM junction at the DLPFC are involved in ASD pathology and associated cognition. The supplementary analysis (Figure S1-4) not only supports the robustness of these findings, but also suggests that provided the equivalence with the present dMRI data quality in combination with multitissue modeling (Jeurissen et al., 2014), FBA could be advantageous to detect small-yet functionally meaningful alterations in voxels containing complex arrangement of axonal compartments, such as at the GM-WM partial volume voxels or cerebral/cerebellar parenchyma. Notably, these findings were only observed using FBA, whereas VBA based on diffusion tensor measures did not yield any significant results. This discrepancy may originate from that tensor-based metrics are prone to the effects of crossing fibers and therefore more susceptible to noise contamination at those brain regions (Tournier et al., 2011).

This study had some limitations. First, the sample had a relatively wide age range. Although our additional age-related tests yielded null results, we cannot exclude residual developmental effects on the current findings. Second, the present sample was male predominant, limiting any formal tests on sex-related differences (Lai et al., 2017). Third, we recruited ASD participants from outpatient clinics, with many participants showing psychiatric comorbidities or/and psychotropic medication uses. On the flip side, this design renders the current results more generalizable to real-world settings. Moreover, there was no difference in ratios of comorbidity and medication utilization between the ASD subgroups. Fourth, our sample size was relatively small, limiting statistical power to detect small between-group effects and paradoxically increasing the risk of inflating effect sizes (Lombardo et al., 2019). Lastly, we did not include intellectual impairment-only without ASD as an additional control condition. Scientific progress will benefit from intellectually-informed recruitment strategies to discover where ASD and intellectual impairment intersect and part ways. 


\section{CONCLUSION}

In sum, we confirmed that changes in anatomical connections linking hemispheres, as well as cerebellum Crus I/II might contribute to autism phenotypes. These ASD-associated alterations appear to be mainly driven by autistic individuals with minimally verbal status and/or intellectual impairment. Across the functional spectrum, autistic severity, nonverbal intelligence and executive function associated with WM fiber-bundle properties in regions where WM pathology based on dMRI are seldom reported. These results highlight that by embracing the inclusion of understudied subpopulations on the spectrum, together with the development of novel neuroimaging methods, we may better reconcile heterogeneity across studies and advance the understanding of the neuropathology of ASD.

\section{ACKNOWLEDGEMENTS}

This study was supported by the Ministry of Science and Technology, Taiwan (105-2628-B-002035-MY3 and 109-2222-E-182-001-MY3). The authors would like to thank all of our participants and their family members for partaking in this study, Ms. Yi-Chun Liu for carrying out the recruitment and data management, Imaging Center for Integrated Body, Mind and Culture Research, NTU for equipment support, and the anonymous reviewers for comments that significantly improved the manuscript.

\section{DECLARATION OF INTERESTS}

All authors have declared that they have no competing or potential conflict of interest or financial interests, which may arise from being named as an author on the manuscript. 


\section{REFERENCES}

Afzali, M., Pieciak, T., Newman, S., Garyfallidis, E., Ozarslan, E., Cheng, H. \& Jones, D. K. (2021). The sensitivity of diffusion MRI to microstructural properties and experimental factors. $J$ Neurosci Methods 347, 108951.

Alvares, G. A., Bebbington, K., Cleary, D., Evans, K., Glasson, E. J., Maybery, M. T., Pillar, S., Uljarević, M., Varcin, K. \& Wray, J. (2020). The misnomer of 'high functioning autism': Intelligence is an imprecise predictor of functional abilities at diagnosis. Autism 24, 221-232.

Ameis, S. H. \& Catani, M. (2015). Altered white matter connectivity as a neural substrate for social impairment in Autism Spectrum Disorder. Cortex 62, 158-81.

American Psychiatric Association (2013). Diagnostic and statistical manual of mental disorders (DSM-5®). American Psychiatric Pub: Arlington, VA.

Anagnostou, E. \& Taylor, M. J. (2011). Review of neuroimaging in autism spectrum disorders: what have we learned and where we go from here. Molecular autism 2, 1-9.

Andersson, J. L. R., Graham, M. S., Drobnjak, I., Zhang, H. \& Campbell, J. (2018). Susceptibility-induced distortion that varies due to motion: Correction in diffusion MR without acquiring additional data. Neuroimage 171, 277-295.

Andersson, J. L. R., Graham, M. S., Drobnjak, I., Zhang, H., Filippini, N. \& Bastiani, M. (2017). Towards a comprehensive framework for movement and distortion correction of diffusion MR images: Within volume movement. Neuroimage 152, 450-466.

Andersson, J. L. R. \& Sotiropoulos, S. N. (2016). An integrated approach to correction for offresonance effects and subject movement in diffusion MR imaging. Neuroimage 125, 1063-1078.

Andrews, D. S., Avino, T. A., Gudbrandsen, M., Daly, E., Marquand, A., Murphy, C. M., Lai, M. C., Lombardo, M. V., Ruigrok, A. N., Williams, S. C., Bullmore, E. T., The Mrc Aims, C., Suckling, J., Baron-Cohen, S., Craig, M. C., Murphy, D. G. \& Ecker, C. (2017). In Vivo Evidence of Reduced Integrity of the Gray-White Matter Boundary in Autism Spectrum Disorder. Cereb Cortex 27, 877-887.

Aoki, Y., Abe, O., Nippashi, Y. \& Yamasue, H. (2013). Comparison of white matter integrity between autism spectrum disorder subjects and typically developing individuals: a meta-analysis of diffusion tensor imaging tractography studies. Mol Autism 4, 25.

Arnold Anteraper, S., Guell, X., D'Mello, A., Joshi, N., Whitfield-Gabrieli, S. \& Joshi, G. (2019). Disrupted Cerebrocerebellar Intrinsic Functional Connectivity in Young Adults with High-Functioning Autism Spectrum Disorder: A Data-Driven, Whole-Brain, High-Temporal Resolution Functional Magnetic Resonance Imaging Study. Brain Connect 9, 48-59.

Basser, P. J., Mattiello, J. \& LeBihan, D. (1994). Estimation of the effective self-diffusion tensor from the NMR spin echo. Journal of Magnetic Resonance, Series B 103, 247-254.

Bedford, S. A., Park, M. T. M., Devenyi, G. A., Tullo, S., Germann, J., Patel, R., Anagnostou, E., Baron-Cohen, S., Bullmore, E. T., Chura, L. R., Craig, M. C., Ecker, C., Floris, D. L., Holt, R. J., Lenroot, R., Lerch, J. P., Lombardo, M. V., Murphy, D. G. M., Raznahan, A., Ruigrok, A. N. V., Smith, E., Spencer, M. D., Suckling, J., Taylor, M. J., Thurm, A., Consortium, M. A., Lai, M. C. \& Chakravarty, M. M. (2020). Large-scale analyses of the relationship between sex, age and intelligence quotient heterogeneity and cortical morphometry in autism spectrum disorder. Mol Psychiatry 25, 614628.

Bernhardt, B. C., Di Martino, A., Valk, S. L. \& Wallace, G. L. (2017). Neuroimaging-Based Phenotyping of the Autism Spectrum. Curr Top Behav Neurosci 30, 341-355.

Buckner, R. L., Krienen, F. M., Castellanos, A., Diaz, J. C. \& Yeo, B. T. (2011). The organization of the human cerebellum estimated by intrinsic functional connectivity. J Neurophysiol 106, 2322-45.

Casanova, M. F. (2014). Autism as a sequence: from heterochronic germinal cell divisions to 
abnormalities of cell migration and cortical dysplasias. Med Hypotheses $\mathbf{8 3}, 32-8$.

Chen, C.-P., Gau, S. S.-F. \& Lee, C.-C. (2019). Toward differential diagnosis of autism spectrum disorder using multimodal behavior descriptors and executive functions. Computer Speech \& Language 56, 17-35.

Chen, Y. L., Shen, L. J. \& Gau, S. S. (2017). The Mandarin version of the Kiddie-Schedule for Affective Disorders and Schizophrenia-Epidemiological version for DSM-5 - A psychometric study. $J$ Formos Med Assoc 116, 671-678.

Crucitti, J., Hyde, C., Enticott, P. G. \& Stokes, M. A. (2020). Are Vermal Lobules VI-VII Smaller in Autism Spectrum Disorder? Cerebellum 19, 617-628.

Curtis, C. E. \& D'Esposito, M. (2003). Persistent activity in the prefrontal cortex during working memory. Trends Cogn Sci 7, 415-423.

D'Mello, A. M., Crocetti, D., Mostofsky, S. H. \& Stoodley, C. J. (2015). Cerebellar gray matter and lobular volumes correlate with core autism symptoms. Neuroimage Clin 7, 631-9.

D'Mello, A. M. \& Stoodley, C. J. (2015). Cerebro-cerebellar circuits in autism spectrum disorder. Front Neurosci 9, 408.

Demetriou, E. A., Lampit, A., Quintana, D. S., Naismith, S. L., Song, Y. J. C., Pye, J. E., Hickie, I. \& Guastella, A. J. (2018). Autism spectrum disorders: a meta-analysis of executive function. Mol Psychiatry 23, 1198-1204.

Den Houting, J. (2019). Neurodiversity: An insider's perspective. SAGE Publications Sage UK: London, England.

Di, X., Azeez, A., Li, X., Haque, E. \& Biswal, B. B. (2018). Disrupted focal white matter integrity in autism spectrum disorder: A voxel-based meta-analysis of diffusion tensor imaging studies. Prog Neuropsychopharmacol Biol Psychiatry 82, 242-248.

Diedrichsen, J. (2006). A spatially unbiased atlas template of the human cerebellum. Neuroimage 33, 127-38.

Dimond, D., Schuetze, M., Smith, R. E., Dhollander, T., Cho, I., Vinette, S., Ten Eycke, K., Lebel, C., McCrimmon, A., Dewey, D., Connelly, A. \& Bray, S. (2019). Reduced White Matter Fiber Density in Autism Spectrum Disorder. Cereb Cortex 29, 1778-1788.

Gau, S. S.-F., Liu, L.-T., Wu, Y.-Y., Chiu, Y.-N. \& Tsai, W.-C. (2013). Psychometric properties of the Chinese version of the social responsiveness scale. Research in autism spectrum disorders 7, 349-360.

Gioia, G. A., Isquith, P. K., Guy, S. C. \& Kenworthy, L. (2000). Behavior rating inventory of executive function: BRIEF. Psychological Assessment Resources: Odessa, FL.

Gotham, K., Pickles, A. \& Lord, C. (2009). Standardizing ADOS scores for a measure of severity in autism spectrum disorders. J Autism Dev Disord 39, 693-705.

Gotham, K., Risi, S., Pickles, A. \& Lord, C. (2007). The Autism Diagnostic Observation Schedule: revised algorithms for improved diagnostic validity. J Autism Dev Disord 37, 613-27.

Guell, X. \& Schmahmann, J. (2020). Cerebellar Functional Anatomy: a Didactic Summary Based on Human fMRI Evidence. Cerebellum 19, 1-5.

Guell, X., Schmahmann, J. D., Gabrieli, J. \& Ghosh, S. S. (2018). Functional gradients of the cerebellum. Elife 7.

Habas, C., Kamdar, N., Nguyen, D., Prater, K., Beckmann, C. F., Menon, V. \& Greicius, M. D. (2009). Distinct cerebellar contributions to intrinsic connectivity networks. J Neurosci 29, 8586-94.

Happe, F. \& Frith, U. (2020). Annual Research Review: Looking back to look forward - changes in the concept of autism and implications for future research. J Child Psychol Psychiatry 61, 218-232.

Honnedevasthana Arun, A., Connelly, A., Smith, R. E. \& Calamante, F. (2021). Characterisation of white matter asymmetries in the healthy human brain using diffusion MRI fixel-based analysis. Neuroimage 225, 117505.

Hus, V. \& Lord, C. (2014). The autism diagnostic observation schedule, module 4: revised algorithm 
and standardized severity scores. J Autism Dev Disord 44, 1996-2012.

Jack, A. \& Pelphrey, K., A. (2017). Annual Research Review: Understudied populations within the autism spectrum - current trends and future directions in neuroimaging research. $J$ Child Psychol Psychiatry 58, 411-435.

Jack, A. \& Pelphrey, K. A. (2015). Neural Correlates of Animacy Attribution Include Neocerebellum in Healthy Adults. Cereb Cortex 25, 4240-7.

Jeurissen, B., Leemans, A., Tournier, J. D., Jones, D. K. \& Sijbers, J. (2013). Investigating the prevalence of complex fiber configurations in white matter tissue with diffusion magnetic resonance imaging. Hum Brain Mapp 34, 2747-66.

Jeurissen, B., Tournier, J. D., Dhollander, T., Connelly, A. \& Sijbers, J. (2014). Multi-tissue constrained spherical deconvolution for improved analysis of multi-shell diffusion MRI data. Neuroimage 103, 411-426.

Just, M. A., Cherkassky, V. L., Keller, T. A., Kana, R. K. \& Minshew, N. J. (2007). Functional and anatomical cortical underconnectivity in autism: evidence from an FMRI study of an executive function task and corpus callosum morphometry. Cereb Cortex 17, 951-61.

Kellner, E., Dhital, B., Kiselev, V. G. \& Reisert, M. (2016). Gibbs-ringing artifact removal based on local subvoxel-shifts. Magn Reson Med 76, 1574-1581.

Kelly, E., Meng, F., Fujita, H., Morgado, F., Kazemi, Y., Rice, L. C., Ren, C., Escamilla, C. O., Gibson, J. M., Sajadi, S., Pendry, R. J., Tan, T., Ellegood, J., Basson, M. A., Blakely, R. D., Dindot, S. V., Golzio, C., Hahn, M. K., Katsanis, N., Robins, D. M., Silverman, J. L., Singh, K. K., Wevrick, R., Taylor, M. J., Hammill, C., Anagnostou, E., Pfeiffer, B. E., Stoodley, C. J., Lerch, J. P., du Lac, S. \& Tsai, P. T. (2020). Regulation of autism-relevant behaviors by cerebellar-prefrontal cortical circuits. Nat Neurosci 23, 1102-1110.

King, M., Hernandez-Castillo, C. R., Poldrack, R. A., Ivry, R. B. \& Diedrichsen, J. (2019). Functional boundaries in the human cerebellum revealed by a multi-domain task battery. Nat Neurosci 22, 1371-1378.

Kirkovski, M., Fuelscher, I., Hyde, C., Donaldson, P. H., Ford, T. C., Rossell, S. L., Fitzgerald, P. B. \& Enticott, P. G. (2020). Fixel Based Analysis Reveals Atypical White Matter Micro- and Macrostructure in Adults With Autism Spectrum Disorder: An Investigation of the Role of Biological Sex. Front Integr Neurosci 14, 40.

Kobayashi, S. (2009). Reward neurophysiology and primate cerebral cortex.

Lai, M. C., Lerch, J. P., Floris, D. L., Ruigrok, A. N., Pohl, A., Lombardo, M. V. \& Baron-Cohen, S. (2017). Imaging sex/gender and autism in the brain: Etiological implications. J Neurosci Res 95, 380-397.

Lin, H. Y., Ni, H. C., Lai, M. C., Tseng, W. I. \& Gau, S. S. (2015). Regional brain volume differences between males with and without autism spectrum disorder are highly age-dependent. Mol Autism 6, 29.

Lin, H. Y., Perry, A., Cocchi, L., Roberts, J. A., Tseng, W. I., Breakspear, M. \& Gau, S. S. (2019). Development of frontoparietal connectivity predicts longitudinal symptom changes in young people with autism spectrum disorder. Transl Psychiatry 9, 86.

Lombardo, M. V., Lai, M. C. \& Baron-Cohen, S. (2019). Big data approaches to decomposing heterogeneity across the autism spectrum. Mol Psychiatry 24, 1435-1450.

Lord, C., Risi, S., Lambrecht, L., Cook, E. H., Jr., Leventhal, B. L., DiLavore, P. C., Pickles, A. \& Rutter, M. (2000). The autism diagnostic observation schedule-generic: a standard measure of social and communication deficits associated with the spectrum of autism. J Autism Dev Disord 30, 205-23.

Lu, T.-H. E. (1993). Adaptive Behavior of the mentally retarded in Taiwan, ROC. Bulletin of Special Education 9, 107-144.

Maenner, M. J., Shaw, K. A., Baio, J., Washington, A., Patrick, M., DiRienzo, M., Christensen, D. L., Wiggins, L. D., Pettygrove, S., Andrews, J. G., Lopez, M., Hudson, A., Baroud, T., Schwenk, 
Y., White, T., Rosenberg, C. R., Lee, L. C., Harrington, R. A., Huston, M., Hewitt, A., Esler, A., Hall-Lande, J., Poynter, J. N., Hallas-Muchow, L., Constantino, J. N., Fitzgerald, R. T., Zahorodny, W., Shenouda, J., Daniels, J. L., Warren, Z., Vehorn, A., Salinas, A., Durkin, M. S. \& Dietz, P. M. (2020). Prevalence of Autism Spectrum Disorder Among Children Aged 8 Years - Autism and Developmental Disabilities Monitoring Network, 11 Sites, United States, 2016. MMWR Surveill Summ 69, 1-12.

Mito, R., Raffelt, D., Dhollander, T., Vaughan, D. N., Tournier, J. D., Salvado, O., Brodtmann, A., Rowe, C. C., Villemagne, V. L. \& Connelly, A. (2018). Fibre-specific white matter reductions in Alzheimer's disease and mild cognitive impairment. Brain 141, 888-902.

Mottron, L. \& Bzdok, D. (2020). Autism spectrum heterogeneity: fact or artifact? Mol Psychiatry 25, 3178-3185.

Olsson, M. B., Holm, A., Westerlund, J., Lundholm Hedvall, Å., Gillberg, C. \& Fernell, E. (2017). Children with borderline intellectual functioning and autism spectrum disorder: developmental trajectories from 4 to 11 years of age. Neuropsychiatr Dis Treat 13, 2519-2526.

Padmanabhan, A., Lynch, C. J., Schaer, M. \& Menon, V. (2017). The Default Mode Network in Autism. Biol Psychiatry Cogn Neurosci Neuroimaging 2, 476-486.

Paul, L. K., Brown, W. S., Adolphs, R., Tyszka, J. M., Richards, L. J., Mukherjee, P. \& Sherr, E. H. (2007). Agenesis of the corpus callosum: genetic, developmental and functional aspects of connectivity. Nat Rev Neurosci 8, 287-99.

Prigge, M. B. D., Bigler, E. D., Travers, B. G., Froehlich, A., Abildskov, T., Anderson, J. S., Alexander, A. L., Lange, N., Lainhart, J. E. \& Zielinski, B. A. (2018). Social Responsiveness Scale (SRS) in Relation to Longitudinal Cortical Thickness Changes in Autism Spectrum Disorder. J Autism Dev Disord 48, 3319-3329.

Raffelt, D., Dhollander, T., Tournier, J.-D., Tabbara, R., Smith, R. E., Pierre, E. \& Connelly, A. (2017a). Bias field correction and intensity normalisation for quantitative analysis of apparent fibre density. In Proc. Intl. Soc. Mag. Reson. Med, p. 3541.

Raffelt, D., Smith, R. E., Ridgway, G. R., Tournier, J. D., Vaughan, D. N., Rose, S., Henderson, R. \& Connelly, A. (2015). Connectivity-based fixel enhancement: Whole-brain statistical analysis of diffusion MRI measures in the presence of crossing fibres. Neuroimage 117, 40-55.

Raffelt, D., Tournier, J. D., Fripp, J., Crozier, S., Connelly, A. \& Salvado, O. (2011). Symmetric diffeomorphic registration of fibre orientation distributions. Neuroimage 56, 1171-80.

Raffelt, D., Tournier, J. D., Smith, R. E., Vaughan, D. N., Jackson, G., Ridgway, G. R. \& Connelly, A. (2017b). Investigating white matter fibre density and morphology using fixel-based analysis. Neuroimage 144, 58-73.

Rodgaard, E. M., Jensen, K., Vergnes, J. N., Soulieres, I. \& Mottron, L. (2019). Temporal Changes in Effect Sizes of Studies Comparing Individuals With and Without Autism: A Meta-analysis. JAMA Psychiatry 76, 1124-1132.

Roid, G. H. \& Miller, L. J. (2002). Leiter-R: Leiter international performance scale-revised. Stoelting: Wood Dale, IL.

Rommelse, N., Buitelaar, J. K. \& Hartman, C. A. (2017). Structural brain imaging correlates of ASD and ADHD across the lifespan: a hypothesis-generating review on developmental ASD-ADHD subtypes. J Neural Transm (Vienna) 124, 259-271.

Rutter, M., Le Couteur, A. \& Lord, C. (2003). Autism diagnostic interview-revised. Los Angeles, CA: Western Psychological Services 29, 30.

Siciliano, L. \& Clausi, S. (2020). Implicit vs. Explicit Emotion Processing in Autism Spectrum Disorders: An Opinion on the Role of the Cerebellum. Front Psychol 11, 96.

Smith, R. E., Tournier, J. D., Calamante, F. \& Connelly, A. (2013). SIFT: Spherical-deconvolution informed filtering of tractograms. Neuroimage 67, 298-312. 
Sparrow, S. S., Balla, D. A., Cicchetti, D. V. \& Harrison, P. L. (1984). Vineland adaptive behavior scales. American Guidance Service: Circle Pines, MN.

Steimke, R., Nomi, J. S., Calhoun, V. D., Stelzel, C., Paschke, L. M., Gaschler, R., Goschke, T., Walter, H. \& Uddin, L. Q. (2017). Salience network dynamics underlying successful resistance of temptation. Soc Cogn Affect Neurosci 12, 1928-1939.

Stoodley, C. J., D'Mello, A. M., Ellegood, J., Jakkamsetti, V., Liu, P., Nebel, M. B., Gibson, J. M., Kelly, E., Meng, F., Cano, C. A., Pascual, J. M., Mostofsky, S. H., Lerch, J. P. \& Tsai, P. T. (2017). Altered cerebellar connectivity in autism and cerebellar-mediated rescue of autism-related behaviors in mice. Nat Neurosci 20, 1744-1751.

Tager-Flusberg, H. \& Kasari, C. (2013). Minimally verbal school-aged children with autism spectrum disorder: the neglected end of the spectrum. Autism Res 6, 468-78.

Taylor, J. L. \& Seltzer, M. M. (2010). Changes in the autism behavioral phenotype during the transition to adulthood. J Autism Dev Disord 40, 1431-46.

Tournier, J. D., Mori, S. \& Leemans, A. (2011). Diffusion tensor imaging and beyond. Magn Reson Med 65, 1532-56.

Tournier, J. D., Smith, R., Raffelt, D., Tabbara, R., Dhollander, T., Pietsch, M., Christiaens, D., Jeurissen, B., Yeh, C. H. \& Connelly, A. (2019). MRtrix3: A fast, flexible and open software framework for medical image processing and visualisation. Neuroimage 202, 116137.

Travers, B. G., Adluru, N., Ennis, C., Tromp do, P. M., Destiche, D., Doran, S., Bigler, E. D., Lange, N., Lainhart, J. E. \& Alexander, A. L. (2012). Diffusion tensor imaging in autism spectrum disorder: a review. Autism Res 5, 289-313.

Tustison, N. J., Avants, B. B., Cook, P. A., Zheng, Y., Egan, A., Yushkevich, P. A. \& Gee, J. C. (2010). N4ITK: improved N3 bias correction. IEEE Trans Med Imaging 29, 1310-20.

Tzourio-Mazoyer, N., Landeau, B., Papathanassiou, D., Crivello, F., Etard, O., Delcroix, N., Mazoyer, B. \& Joliot, M. (2002). Automated anatomical labeling of activations in SPM using a macroscopic anatomical parcellation of the MNI MRI single-subject brain. Neuroimage 15, 273-89.

Uddin, L. Q. (2015). Salience processing and insular cortical function and dysfunction. Nat Rev Neurosci 16, 55-61.

van der Heijden, M. E., Gill, J. S. \& Sillitoe, R. V. (2021). Abnormal Cerebellar Development in Autism Spectrum Disorders. Dev Neurosci, 1-10.

Veraart, J., Fieremans, E. \& Novikov, D. S. (2016). Diffusion MRI noise mapping using random matrix theory. Magn Reson Med 76, 1582-1593.

Wasserthal, J., Neher, P. \& Maier-Hein, K. H. (2018). TractSeg-Fast and accurate white matter tract segmentation. NeuroImage 183, 239-253.

Wechsler, D. (2008). Wechsler adult intelligence scale - Fourth Edition (WAIS-IV). Pearson: San Antonio, TX.

Wechsler, D. \& Kodama, H. (2003). Wechsler intelligence scale for children (4th ed.). The Psychological corporation: San Antonio, TX.

Xu, J., Moeller, S., Auerbach, E. J., Strupp, J., Smith, S. M., Feinberg, D. A., Yacoub, E. \& Ugurbil, K. (2013). Evaluation of slice accelerations using multiband echo planar imaging at $3 \mathrm{~T}$. Neuroimage 83, 991-1001.

Yeo, B. T., Krienen, F. M., Sepulcre, J., Sabuncu, M. R., Lashkari, D., Hollinshead, M., Roffman, J. L., Smoller, J. W., Zöllei, L., Polimeni, J. R., Fischl, B., Liu, H. \& Buckner, R. L. (2011). The organization of the human cerebral cortex estimated by intrinsic functional connectivity. $J$ Neurophysiol 106, 1125-65.

Yuan, Z., Qin, W., Wang, D., Jiang, T., Zhang, Y. \& Yu, C. (2012). The salience network contributes to an individual's fluid reasoning capacity. Behav Brain Res 229, 384-90.

Zhang, Z., Peng, P. \& Zhang, D. (2020). Executive Function in High-Functioning Autism Spectrum 
bioRxiv preprint doi: https://doi.org/10.1101/2021.10.11.464005; this version posted October 13, 2021. The copyright holder for this preprint (which was not certified by peer review) is the author/funder, who has granted bioRxiv a license to display the preprint in perpetuity. It is made available under aCC-BY-ND 4.0 International license.

Disorder: A Meta-analysis of fMRI Studies. Journal of Autism \& Developmental Disorders $\mathbf{5 0 .}$ 


\section{Supplementary Material}

\section{White matter microstructural and morphometric alterations in autism: the implications of intellectual and verbal capabilities}

Chun-Hung Yeh, Rung-Yu Tseng, Hsing-Chang Ni, Luca Cocchi, Jung-Chi Chang, Mei-Yun Hsu, EnNien Tu, Yu-Yu Wu, Tai-Li Chou, Susan Shur-Fen Gau, Hsiang-Yuan Lin

\section{A. Supplementary Table S1. Comorbidity and Medication Use for ASD-IA \& ASD-II}

\begin{tabular}{|c|c|c|}
\hline \multirow[b]{2}{*}{ Comorbidity } & \multirow[t]{2}{*}{ ASD-IA $(n=34)$} & \multirow[t]{2}{*}{ ASD- $\|(n=31)$} \\
\hline & & \\
\hline No comorbidity & 8 & 7 \\
\hline Comorbid ADHD & 20 & 18 \\
\hline Comorbid Anxiety disorder & $\begin{array}{l}8 \text { ( } 5 \text { social anxiety disorder; } 1 \\
\text { history of mild agoraphobia; } \\
1 \text { height phobia) }\end{array}$ & $\begin{array}{l}9 \text { ( } 4 \text { specific phobia; } 4 \text { social } \\
\text { anxiety disorder; } 1 \text { selective } \\
\text { mutism) }\end{array}$ \\
\hline Comorbid tic disorder & 3 & 5 (2 Tourette syndrome) \\
\hline Comorbid OCD & 1 & 1 \\
\hline Comorbid learning disorder & 1 (writing/reading disorder) & $\mathrm{N} / \mathrm{A}$ \\
\hline Comorbid depressive disorder & $\begin{array}{l}3 \text { ( } 1 \text { history of depression; } 1 \\
\text { dysthymic disorder \& mild } \\
\text { MDD) }\end{array}$ & $\mathrm{N} / \mathrm{A}$ \\
\hline Other comorbidities & 1 gender dysphoria & 2 epilepsy (well controlled) \\
\hline & 1 ODD & 1 ODD \\
\hline \multicolumn{3}{|l|}{ Medications } \\
\hline Methylphenidate & 13 & 6 \\
\hline Antidepressant & 2 (1 sertraline; 1 fluoxetine) & 1 (sertraline) \\
\hline Valproic acid (mood stabilizer) & $\mathrm{N} / \mathrm{A}$ & 2 \\
\hline Antipsychotic & $\mathrm{N} / \mathrm{A}$ & $\mathrm{N} / \mathrm{A}$ \\
\hline
\end{tabular}

Acronym - ADHD: Attention Deficit/Hyperactivity Disorder; MDD: Major Depressive Disorder; OCD: Obsessive Compulsive Disorder; ODD: Oppositional Defiant Disorder. 


\section{B. Mean fiber density of the splenium of corpus callosum across groups}

The outcomes of the categorical analysis shown in Figure 1 demonstrated that the significant differences of FBA metrics were found at: TDC > ASD-Whole, TDC > ASD-II, and ASD-II-Only > ASD-MV. When knowing TDC > ASD-Whole, one would expect that TDC should also have greater fixel metrics (e.g. FD) than any ASD subgroups. Thus, the results of no significant differences between TDC and ASD-IA might seem to be contradictory to such expectations. The purpose of this section is to attempt to explain the rationale behind the relevant findings.

Figure S1 shows the complementary outcomes to Figure 1, from which a mask containing "significant fixels" at the splenium of the corpus callosum was derived first. For each (sub)group, the mean and standard deviation of the FD metric within the fixel mask was then computed for each participant, yielding the group distribution of FD. Consistent with Figure 1, TDC showed significantly higher mean FD than ASD-Whole. In addition, divided from ASD-Whole, the results suggest that the distribution of ASD-IA "shifted" toward TDC, thus yielding non-significant differences between TDC and ASD-IA.

Likewise, Figure S1 also explains why there were no differences between ASD-IA and ASD-II: When separated from ASD-Whole, the distribution of ASD-II indeed "shifted" oppositely with ASDIA. However, although both subgroups had significant differences in their clinical features (Table 1), the alteration in mean FD did not reach the statistical significance. Nevertheless, such a shift toward reduced mean FD resulted in a significantly lower value of ASD-II as compared to TDC, which was consistent with the outcomes of FBA shown in Figure 1.

Combining Figures 1 and S1 therefore leads us to the conclusion that fiber-specific alterations are driven by those combined with II and/or MV, and that it is imperative to include these populations in ASD research to avoid the selection bias. 


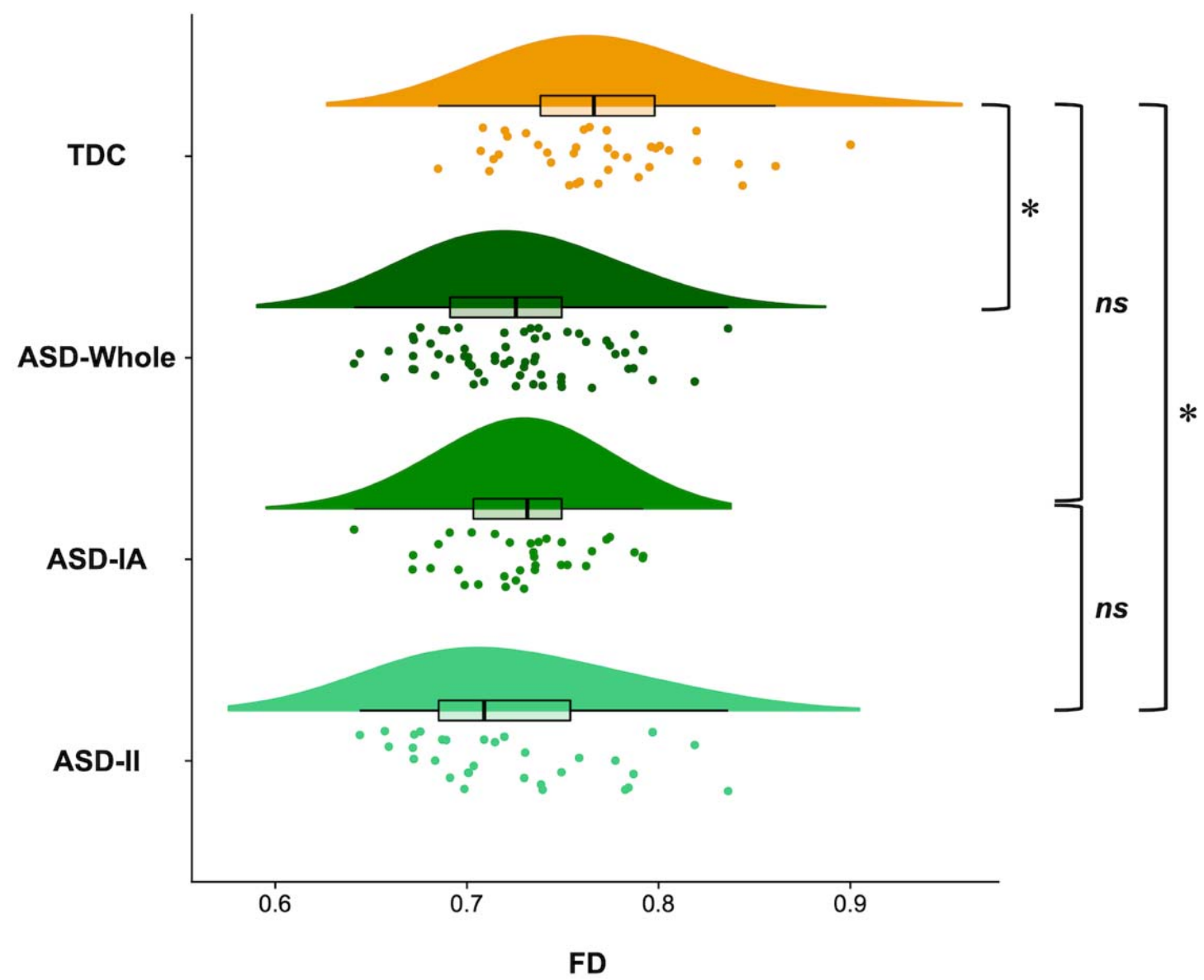

Figure S1: Raincloud plots showing the distribution of per participant's mean fiber density (FD) metric in each (sub)group. FD values were extracted from fixels that showed significant reductions in FD at the splenium of the corpus callosum, which was defined by the spatial overlap between the outcome of TDC vs. ASD-Whole and TDC vs. ASD-II. “*” indicates significant differences of the distribution; " $n s$ " denotes that the differences between groups are non-significant.

\section{FBA at different thresholds of FOD peak amplitudes}

The threshold of the FOD peak value defines the minimum amplitude of an FOD lobe to be regarded as a fixel. Hence, lowering the threshold will include small fixels potentially representing a small fraction of fibers presenting in a voxel, at the price of increasing false positive fixels that could come from the effect of noise contamination. Likewise, increasing the threshold will make the FBA focus only on large fixels or major WM fiber bundles, it would however have a detrimental effect as 
the contribution of fixels below the threshold to the DWI signal will be completely discarded. The default FOD cutoff in MRtrix3 was set to 0.06 empirically, we therefore provide the results of complementary FBA in this section, in which we adjust the value to 0.04 / 0.08 / 0.10 to ensure that the findings in the current study were not determined by the choice of the threshold. Overall, the FBA outcomes remain largely consistent following the adjustment of the FOD cutoff value. Despite some expected variations in the spatial extent of the significant fixels, no additional anatomical regions were identified.

Figure S2 shows the results of categorical comparisons under the FOD cutoff values of 0.04 / 0.08 / 0.10. Comparing with the default threshold of 0.06 shown in Figure 1, only one brain region where the group differences became non-significant: In TDC versus ASD-II, no significant difference in FD at the posterior CC was detected under the cutoff value of 0.10 . 


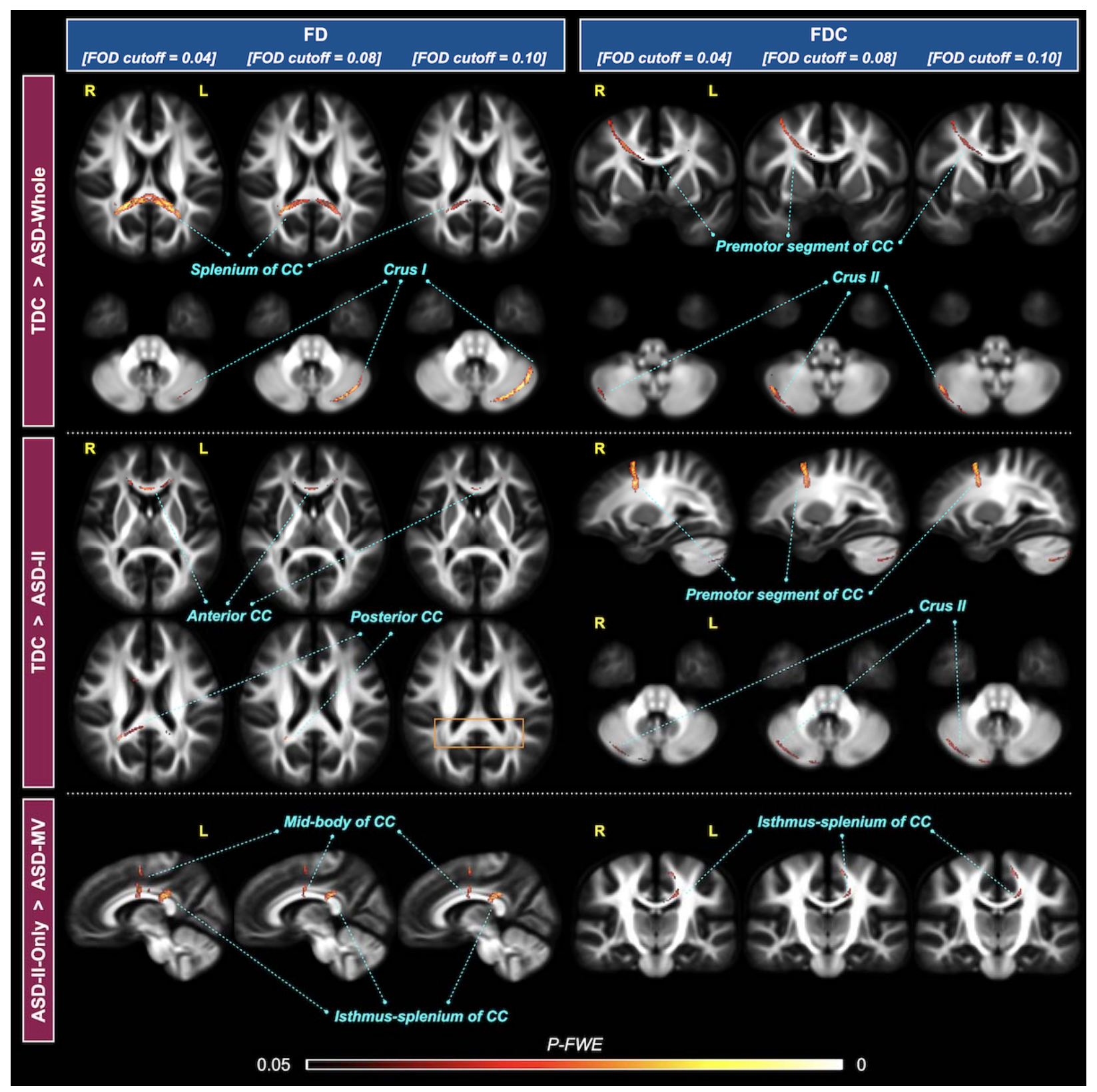

Figure S2: Results of categorical comparisons using an FOD cutoff threshold of 0.04, 0.08, and 0.10. Different thresholds were listed from left to right, for the FD (left column) and FDC (right column) metric. Significant fixels are colored by $P-F W E$ and overlaid on the FOD template. Upper block - TDC $>$ ASD-Whole; middle block - TDC > ASD-II; bottom block - ASD-II-Only > ASD-MV. The rectangle colored in orange indicates the missing significance comparing to the original results under the FOD cutoff of 0.06 in Figure 1 of the main content. 
bioRxiv preprint doi: https://doi.org/10.1101/2021.10.11.464005; this version posted October 13, 2021. The copyright holder for this preprint (which was not certified by peer review) is the author/funder, who has granted bioRxiv a license to display the preprint in perpetuity. It is made available under aCC-BY-ND 4.0 International license.

Figure S3 shows that the dimensional brain-behavior analysis under different FOD cutoff values has a similar pattern with the results shown in Figures 2 and 3.

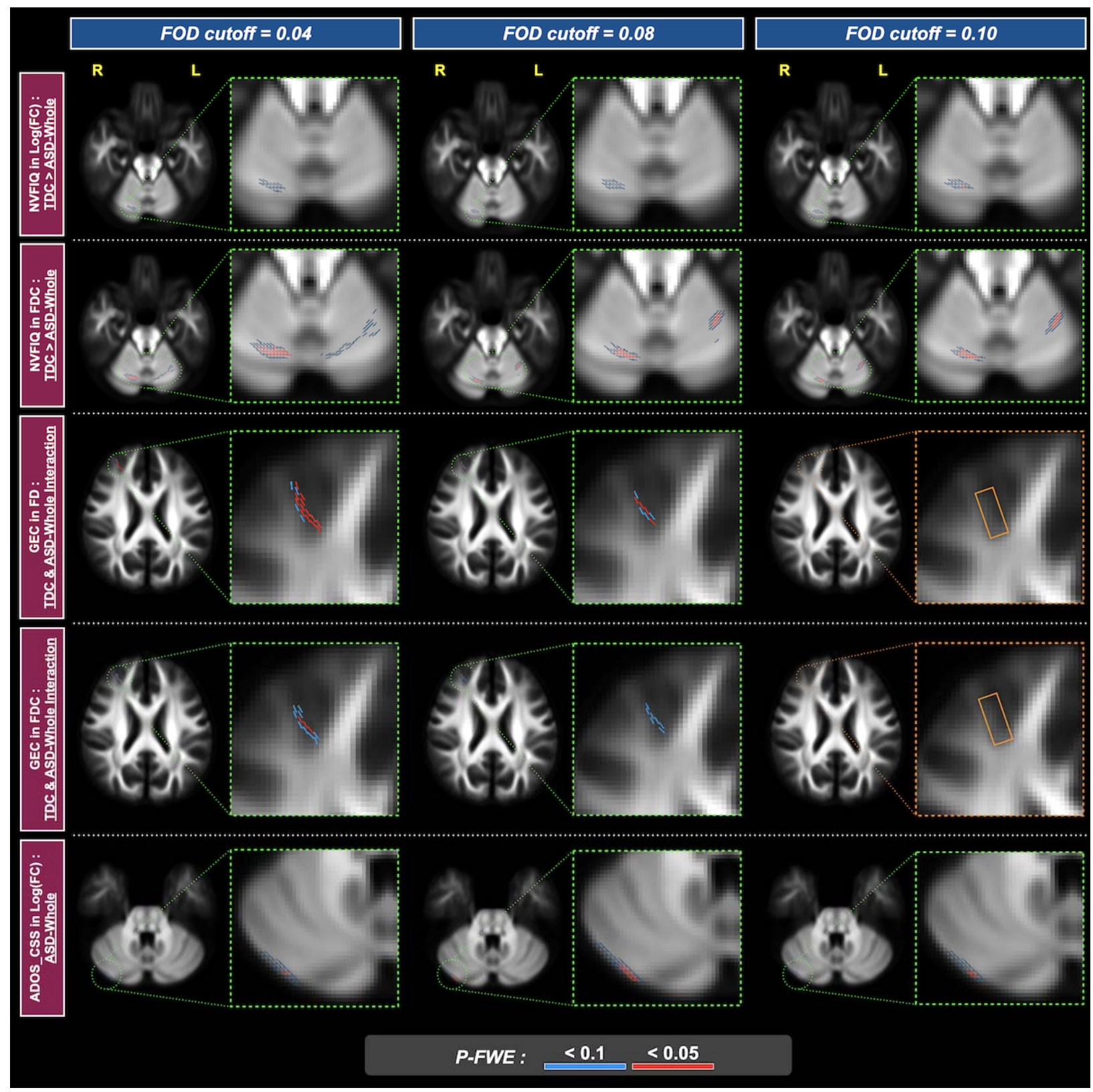

Figure S3: Results of dimensional brain-behavior analysis using an FOD cutoff threshold of 0.04 (left column), 0.08 (middle column), and 0.10 (right column). Each row illustrates the correlations between a fixel metric and a behavior score, in comparison to the outcomes under the FOD cutoff of 0.06 shown in Figures 2 and 3 of the main content. The rectangle colored in orange indicates the missing significance comparing to Figure 3. Fixels are colored in red for $P-F W E<0.05$; fixels colored in blue indicate $P-F W E<0.1$ and are used to assist identification of the associated brain structure. 
Interestingly, changing the FOD threshold appears to alter the FBA results oppositely in the cerebrum and cerebellum, as observed from both the categorical comparisons and dimensional analysis above. The application of a higher FOD cutoff value results in a reduced significance level in the cerebrum but an enhanced statistics in the cerebellum, either based on the FD or FDC metric. The explanation for this contrary tendency is not straightforward, as such a tendency might be dependent on the spatial location and study-specific. Ideally, one could consider using a lower FOD threshold, given sufficient quality of dMRI data. The outcomes provided in this section suggest that the default FOD cutoff of 0.06 used in this study might be the right balance for investigating both the cerebrum and cerebellum.

\section{FBA at a significance level of $P-F W E=0.01$}

This section provides the results of using a more stringent statistical level of $P-F W E<0.01$, with all other processing procedures and parameters identical to those in the main article. Supplementary Figures S4-5 show the results for the categorical comparisons and dimensional analysis, respectively. 


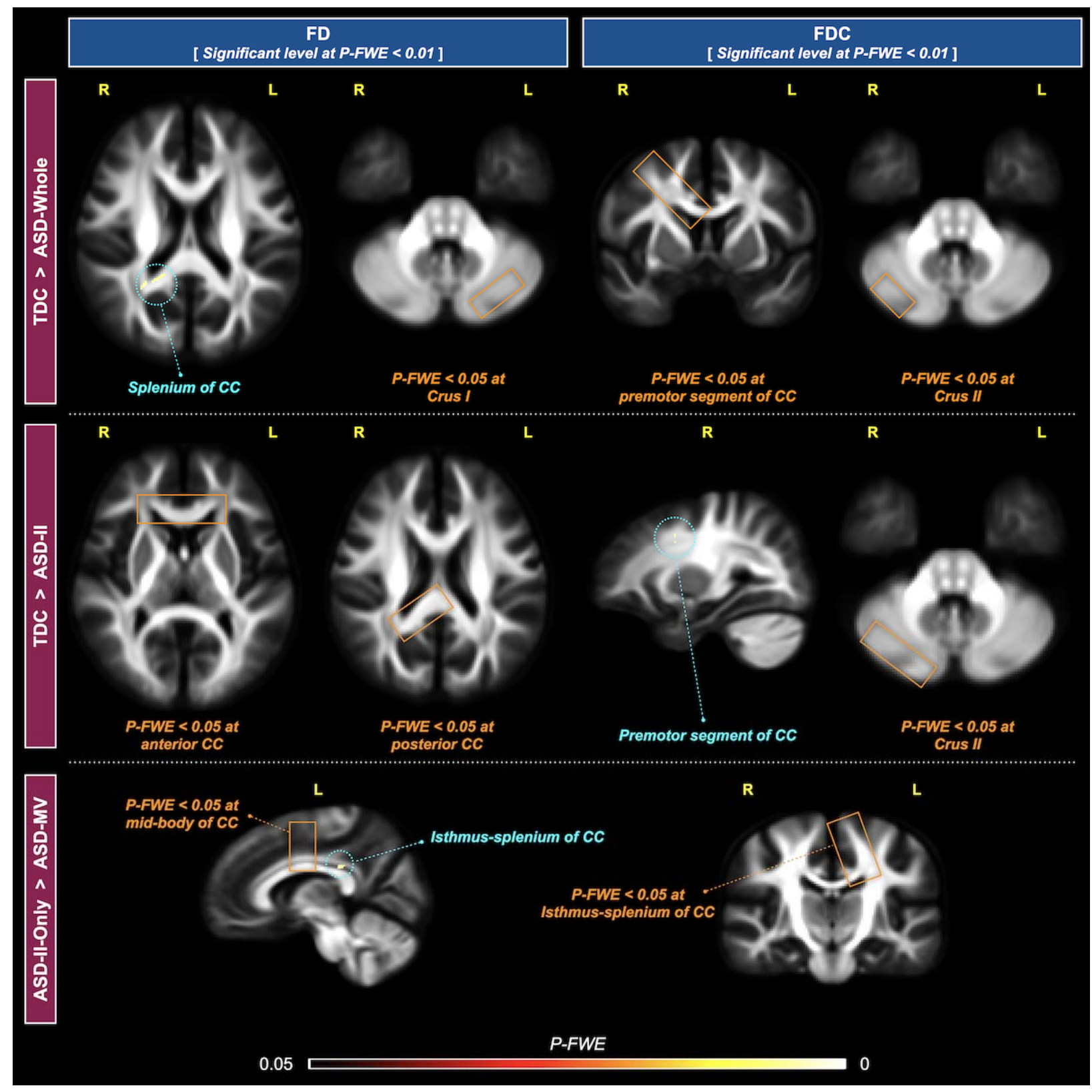

Figure S4: Results for categorical comparisons under a statistical threshold of $P-F W E<0.01$. Left column - metric FD; right column - metric FDC. Upper block - TDC > ASD-Whole; middle block TDC > ASD-II; bottom block - ASD-II-Only > ASD-MV. Fixels within blue dashed circles reached the significance level of $P-F W E<0.01$. The rectangles colored in orange highlight brain regions where fixels reached $P-F W E<0.05$ but did not pass the statistical testing using $P-F W E<0.01$. 


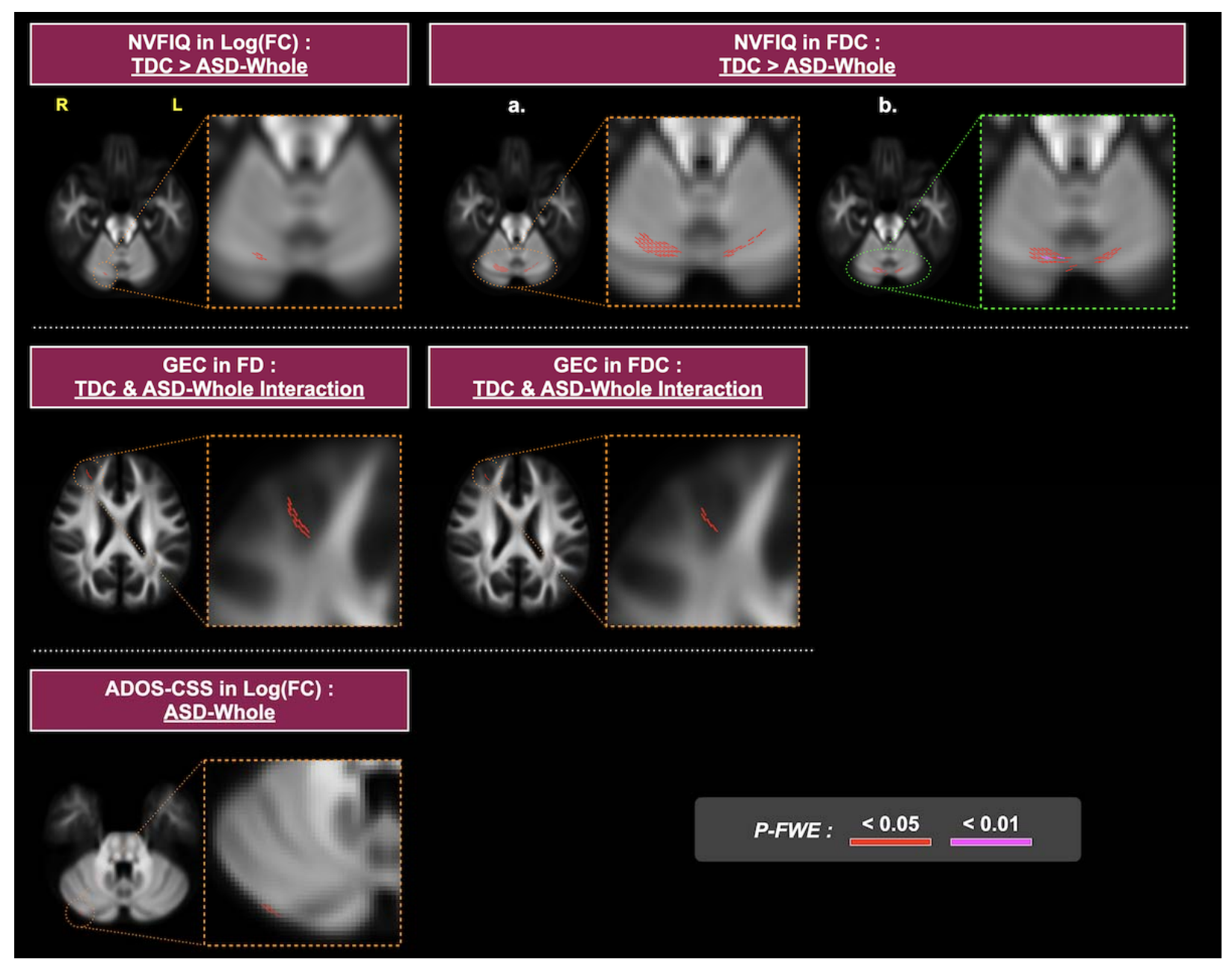

Figure S5: Results of dimensional analysis under a statistical threshold of $P-F W E<0.01$. Upper row NVFIQ: The only significance under this more restricted threshold is in the positive correlation of NVFIQ and FDC. Panel a shows the same slice in main content, where no significant results appear at $P-F W E<0.01$. Panel $\mathrm{b}$ is the result at one slice below Panel a, where the significance under $P-F W E<$ 0.01 are observed. Middle row - GEC: no fixels reach $P-F W E<0.01$. Bottom row - ADOS-CSS: no fixels reach $P-F W E<0.01$. Fixels are colored in magenta for $P-F W E<0.01$; fixels colored in red indicate $P-F W E<0.05$ and are used to assist identification of the associated brain structure.

\section{E. Voxel-based analysis based on diffusion tensor model}

This section provides the results obtained from the same study cohort using the conventional voxel-based analysis (VBA) based on the diffusion tensor metrics to benchmark against the present FBA outcomes. To this end, FA and MD maps were computed for each participant using the $b=0$ and 
b=1000 DWI volumes (90 diffusion gradient directions) extracted from the multi-shell DWI data at the preprocessed level. The FA maps of all TDC and ASD-Whole participants were used to generate a study-specific FA template. The FA and MD maps of each participant were then transformed to the template space via applying the transformation field obtained from the image registration between individual's FA map and the template. A Gaussian kernel of 6-mm full width at half-maximum in size was applied to smooth the transformed FA and MD maps. The whole-brain statistical analysis was performed using GLM, with the threshold-free cluster enhancement applied. The nuisance variables included participant's sex, age, medication, and relative RMS. All analyses including both the categorical and dimensional analysis as in FBA were performed for VBA. Nonparametric testing was performed using 5,000 permutations, and the significance level was defined at $P-F W E<0.05$. All the processing steps above were done using MRtrix3.

Supplementary Figure S6 shows the voxels/regions that reached statistical significance $(P-F W E<$ 0.05). For the categorical comparisons, the TDC group has higher FA than the ASD-Whole at a cluster of voxels within the third ventricle. The dimensional brain-behavior analysis showed that there was a positive correlation between FA and GEC at a cluster that was also located in the brain ventricle. Both results however cannot draw a meaningful interpretation. All the results reported from FBA in the present study were not found using either FA- or MD-based VBA. 


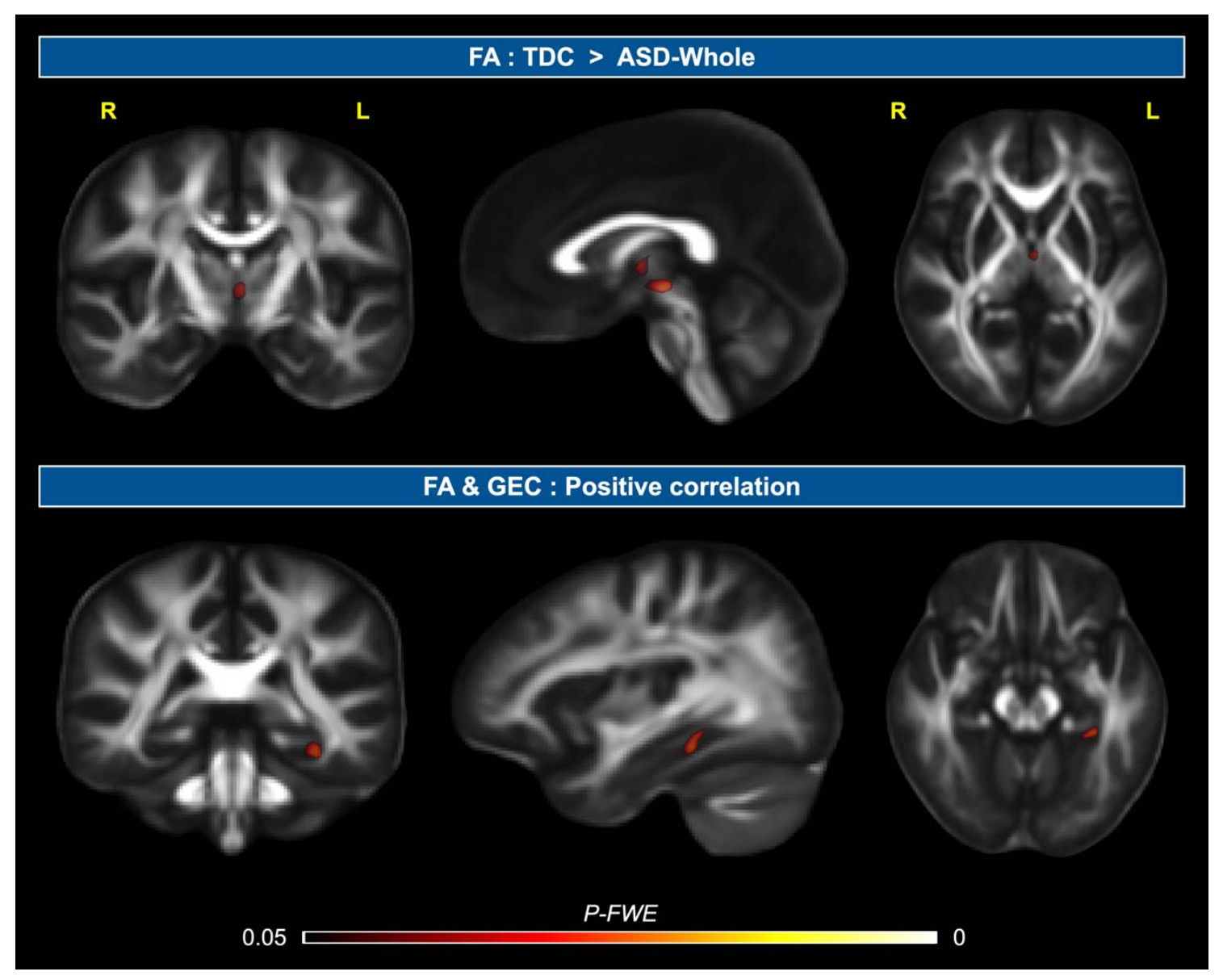

Figure S6: Results of VBA using the FA and MD metrics of the diffusion tensor model. Voxels that reached statistical significance $(P-F W E<0.05)$ are colored by the $P-F W E$ and overlaid on the group FA template images in three orthogonal planes. Upper row - the cluster where TDC have greater FA than ASD-Whole. Bottom row - the cluster where GEC positively correlates with FA. 